\title{
Statistical Learning Algorithms Applied to Automobile Insurance Ratemaking
}

\author{
C. Dugas, Y. Bengio, N. Chapados, P. Vincent, \\ G. Denoncourt and C. Fournier
}

The chapter will start from a description of the fundamentals of statistical learning algorithms and highlight how its basic tenets and methodologies differ from those generally followed by actuaries and econometricians. The main premise is that reality is too complex to be captured with a single unifying model, although some aspects may be well approximated by models. Therefore the statistical learning approach does not presume that reality is perfectly captured by a model, or at least tries to minimize the assumptions about the true generating distribution of the data. The approach is empirical: good models will be distinguished from poor models by comparing their predictive power and explanatory power on new data. At this point it is interesting to consider that choosing among models may be guided by two different objectives, which sometimes yield to different answers: an operational objective (which model will yield to the best decisions/predictions on new data), or a "modeling" objective (which model better describes the true underlying nature of the data). We will show an example in which the two approaches yield to different statistical tests and the operational approach yields to more conservative decisions (chooses simpler models). Another example of the difference between the two approaches is illustrated by the case of ridge regression: there is a regularized (biased) regression that brings better out-of-sample expected predictions than the maximum likelihood (unbiased) estimator. This example will be used to illustrate the famous bias-variance dilemma that is so pervasive in statistical learning algorithms. 
The above discussion and introduction to the principles of statistical learning will naturally yield to the issue of methodology. We will describe and justify the main methodological tools of the statistical learning approach for selecting and comparing models, either based on theoretical bounds or on resampling techniques (such as the cross-validation and bootstrap techniques). A special section on the particular (and rarely discussed) issue of non-stationary data will explain how the above resampling methods can be generalized to data whose distribution varies over time, which is the case with insurance data. In order to evaluate and compare models, one needs to build statistical tools to evaluate the uncertainty in the measurements of out-of-sample performance (due to finite data and non-stationarity).

We applied the principles and methodology described above in a research contract we recently conducted for a large North American automobile insurer. This study was the most exhaustive ever undertaken by this particular insurer and lasted over an entire year. We analyzed the discriminative power of each variable used for ratemaking. We analyzed the performance of several statistical learning algorithms within five broad categories: Linear Regressions, GLMs, Decision Trees, Neural Networks and Support Vector Machines. We present the main results of this study.

We qualitatively compare models and show how Neural Networks can represent high order nonlinear dependencies with a small number of parameters, each of which is estimated on a large proportion of the data thus, yielding low variance. We thoroughly explain the purpose of the nonlinear sigmoidal transforms which are at the very heart of Neural Networks' performances. The main numerical result is a statistically significant reduction in the out-of-sample mean-squared error using the Neural Network model.

In some provinces and states, better risk discrimination, if not directly implemented because of market share concerns or legislative constraints, can also be used for the purpose of choosing the risks 
to be sent to "risk-sharing pools". According to these plans, insurers choose a portion of their book of business which they ceed to the pool. Losses (seldom gains) are shared by participants and/or insurers doing business in the province or state of the plan. Since the selection of risks to be sent to the pool bears no effect on market share (the insured is unaware of the process) and legislation is generally looser than that of ratemaking, highly discriminative statistical learning algorithms such as Neural Networks can be very profitably used to identify those most underpriced risks that should be ceeded to the pool. We compare Generalized Linear Models to our Neural Network based model with respect to their risk-sharing pool performance.

\section{Introduction}

Ratemaking is one of the main mathematical problems faced by actuaries. They must first estimate how much each insurance contract is expected to cost. This conditional expected claim amount is called the pure premium and it is the basis of the gross premium charged to the insured. This expected value is conditioned on information available about the insured and about the contract, which we call the input profile.

Automobile insurance ratemaking is a complex task for many reasons. First of all, many factors are relevant. Taking account of each of them individually, i.e., making independence assumptions, can be hurtful (Bailey \& Simon 1960). Taking account of all interactions is intractable and is sometimes referred to as the curse of dimensionality (Bellman 1957). In practice, actuarial judgment is used to discover the most relevant of these interactions and feed them explicitly to the model. Neural networks, on the other hand, are well-known for their ability to represent high order nonlinear interactions with a small number of parameters, i.e., they can automatically detect those most relevant interactions between variables (Rumelhart, Hinton \& Williams 1986). We explain how and why in section 5. 
A second difficulty comes from the distribution of claims: asymmetric with fat tails with a large majority of zeros and a few unreliable and very large values, i.e., an asymmetric heavy tail extending out toward high positive values. Modeling data with such a distribution is essentially difficult because outliers, which are sampled from the tail of the distribution, have a strong influence on parameter estimation. When the distribution is symmetric around the mean, the problems caused by outliers can be reduced using robust estimation techniques (Huber 1982, Hampel, Ronchetti, Rousseeuw \& Stahel 1986, Rousseeuw \& Leroy 1987) which basically intend to ignore or down-weight outliers. Note that these techniques do not work for an asymmetric distribution: most outliers are on the same side of the mean, so downweighting them introduces a strong bias on its estimation: the conditional expectation would be systematically underestimated. Recent developments for dealing with asymmetric heavy-tail distributions have been made (Takeuchi, Bengio \& Kanamori 2002).

The third difficulty is due to the non-stationary nature of the relationship between explanatory variables and the expected claim amount. This has an important effect on the methodology to use, in particular with respect to the task of model selection. We describe our methodology in section 4 .

Fourth, from year to year, the general level of claims may fluctuate heavily, in particular in states and provinces where winter plays an important role in the frequency and severity of accidents. The growth of the economy and the price of gas can also affect these figures.

Fifth, one needs sufficient computational power to develop models: we had access to a large database of $\approx 8 \times 10^{6}$ records, and the training effort and numerical stability of some algorithms can be burdensome for such a large number of training examples.

Sixth, the data may be of poor quality. In particular, there may be missing fields for many records. An actuary could systematically dis- 
card incomplete records but this leads to loss of information. Also, this strategy could induce a bias if the absence of a data is not random but rather correlated to some particular feature which affects the level of risk. Alternatively one could choose among known techniques for dealing with missing values (Dempster, Laird \& Rubin 1977, Ghahramani \& Jordan 1994, Bengio \& Gingras 1996).

Seventh, once the pure premiums have been established the actuary must properly allocate expenses and a reserve for profit among the different contracts in order to obtain the gross premium level that will be charged to the insureds. Finally, an actuary must account for competitive concerns: his company's strategic goals, other insurers' rate changes, projected renewal rates and market elasticity.

In this chapter, we address the task of setting an appropriate pure premium level for each contract, i.e., difficulties one through four as described above. Our goal is to compare different models with respect to their performance in that regard, i.e., how well they are able to forecast the claim level associated to each contract. We chose several models within five broad categories: Linear Regressions, Generalized Linear Models (McCullagh \& Nelder 1989), Decision Trees (Kass 1980), Neural Networks and Support Vector Machines (Vapnik 1998).

The rest of the chapter is organized as follows: in section 2 we introduce the reader to some of the fundamental principles underlying statistical machine learning, compare them to those that govern more traditional statistical approaches and give some examples. Then, we describe usual candidate mathematical criteria that lead to insurance premium estimation in section 3. Statistical learning methodologies are described in section 4, with an emphasis on the one that was used within the course of the study. This is followed in section 5 by a review of the statistical learning algorithms that we considered, including our best-performing mixture of positive-output Neural Networks. We describe experimental results w.r.t. ratemaking in section 6 . In section 
7, we compare two models on the task of identifying the risks to be sent to a risk sharing pool facility. In view of these results we conclude with an examination of the prospects for applying statistical learning algorithms to insurance modeling in section 8 .

\section{Concepts of Statistical Learning Theory}

Statistical inference is concerned with the following: Given a collection of empirical data originating from some functional dependency, provide answers to questions that could be answered if that dependency were known. Although elements of statistical inference have existed for more that 200 years (Gauss, Laplace), it is within the last century that the development of methods and their formal analysis began.

Fisher developped the framework of parametric statistics and suggested one method of approximating the unknown parameter values of a particular model: maximum likelihood. Glivenko, Cantelli and Kolmogorov used a more general approach as they proved that the empirical distribution function converges exponentially to the actual distribution function. Most importantly, this result is independent of the unknown actual distribution function. These two fundamental results can be seen as the seeds of two philosophically diverging frameworks of statistical inference.

The goal of the first approach is to identify the data generating process. For the purpose of this modelling goal, one must have sufficient knowledge of the physical laws that govern the process in order to build a corresponding model. The essence of that branch of statistical inference is therefore to estimate the unknown parameter values of a (presumably) known model, using the available collection of empirical data, then to devise statistical tests that lead to rejection or not of the model (or of some of its parameters). For the purpose 
of parameter estimation, one often adopts the maximum likelihood method, which enjoys attractive asymptotic properties.

On the other hand, according to the second approach, one merely attemps to predict properties of future data, based on the already given observed data. The belief is that the reality of the process is too complex to be identified and captured in a single unifying model. In particular, multivariate processes are faced with the problem that R. Bellman called "the curse of dimensionality" since the number of combinations of variable values increases exponentially with the dimensionality (the number of explanatory variables) of the problem. In real-life problems, such as automobile insurance where one considers dozens of variables, the belief that one can truly identify the generating process looks naive. The goal of the second approach therefore less ambitious: given a collection of data and a set of candidate functions, find an approximation to the observed unknown process (or a function that can answer the desired questions about the data, such as future conditional expectation) in order to obtain the best performance on predictive tasks, on new data. In the face of this operational goal, statistical inference had to evolve.

In the 1960's, P. Huber developed the robust approach to parametric statistics. In the 1970's, in order to widen the sets of functions to choose from, J. Nedler and R. Wedernburn developed the Generalized Linear Models which have lately become increasingly popular in the actuarial community. The availability of wider sets of functions lead to the problem of model selection. In the 1980's, L. Breiman P. Huber and J. Friedman started to consider special types of function, nonlinear in their parameters and with fewer distributional assumptions (in particular Decision Trees and Neural Networks) and also developed the regularization method as an alternative to the maximum likelihood method, one better suited to the operational goal of achieving the best out-of-sample predictive performance.

A branch of statistical learning (or machine learning) is mainly con- 
cerned with the development of proper refinements of the regularization and model selection methods in order to improve the predictive ability of algorithms. This ability is often referred to as generalization, since the algorithms are allowed to generalize from the observed training data to new data. One crucial element of the evaluation of the generalization ability of a particular model is the measurement of the predictive performance results on out-of-sample data, i.e., using a collection of data, disjoint from the in-sample data that has already been used for model parameter estimation. In the case of automobile insurance, where data is not i.i.d. but rather bears a sequential structure with potential non-stationarities (changes in the underlying generating process w.r.t. its explanatory variables), this requirement leads to the particular methodology of sequential validation which we shall explain in detail in section 4.

\subsection{Hypothesis Testing: an example}

Let us illustrate these concepts with a simple example where the two approaches yield to different statistical tests, thus possibly different conclusions. Consider the classical statistical linear regression tests for deciding to keep a coefficient. Let the relation $E[Y \mid x]=\alpha+\beta x$ hold, with $\operatorname{Var}[Y \mid x]=\sigma^{2}$ the output noise. The classical statistical test for rejecting the input $X$ (i.e. setting the $\beta$ coefficient to 0 in the model) is based on the null hypothesis $\beta=0$. In this context, however, one should distinguish two questions: (1) is $\beta$ really equal to zero? (this is what the above classical test tries to determine), or (2) would choosing $\hat{\beta}=0$ yield better or worse out-of-sample expected generalization than choosing $\hat{\beta}$ that minimizes the in-sample error? Let us define the generalization error as the expected out-of-sample error:

$$
E S E=E\left[(Y-(\hat{\alpha}+\hat{\beta} X))^{2}\right]
$$

As shown in (Gingras, Bengio \& Nadeau 2000), if one is more interested in generalization error, then one should not use the classical 
test, but rather choose an out-of-sample test. Examples of unbiased out-of-sample tests are shown in (Nadeau \& Bengio 2000).

In particular one can show that if the true $\beta$ is such that the signal to noise ratio $\frac{\beta^{2}}{\sigma^{2}}$ is less than (greater than) some positive threshold value $\theta$, then setting $\hat{\beta}$ to zero (the in-sample estimator) will generalize better. When the data set has input values $\left(X_{1}, X_{2}, \ldots, X_{n}\right)$, and writing the input average $\bar{X}=\frac{1}{N} \sum_{i=1}^{n} X_{i}$, the threshold value is

$$
\theta=\frac{E\left[\frac{(X-\bar{X})^{2}}{\sum_{i}\left(X_{i}-\bar{X}\right)^{2}}\right]}{E\left[(X-\bar{X})^{2}\right]},
$$

where $X$ is an out-of-sample example (not one of the training set $X_{i}$ ). Thus the out-of-sample tests tell us to reject a parameter when the signal-to-noise for that parameter is too small, even if the "true" value of that parameter is non-zero. This is because by trying to estimate that parameter from too little data, one is bound to worsen expected generalization error.

What empirical tests show in (Gingras et al. 2000) is that if one is really interested in knowing whether $\beta=0$ in the above example, then one should really use the classical statistical test rather than a generalization error statistic, because for that null hypothesis the former has greater power than the latter. On the other hand, if one is really interested in out-of-sample predictive performance, one should use a generalization error test, because the classical test is liberal (i.e. it keeps a non-zero $\beta$ too often), which can be very dangerous in applications.

Finally, although the difference between the two types of statistics becomes small as $n$ goes to infinity ( $\theta$ above goes to zero), it should be noted that in insurance applications with many input variables, the "small-sample" effect is not negligible, for two reasons:

1. when the number of discrete variables is large, and we want to 
take into account their joint effects, the number of possible combinations of their values is very large; thus there is really very little data to decide whether a parameter associated to a particular combination is useful or not,

2. when the claims distribution is highly asymmetric (i.e. the mean is far from the median), the rare large claims can have a very strong effect on the parameter estimation (i.e. the noise is strong), which increases the discrepency between the conclusions reached with the in-sample and out-of-sample statistics.

In the above analysis, there is another reason for prefering the operational approach in practical applications: the out-of-sample statistical tests do not require any assumption on the form of the underlying distribution ${ }^{1}$. In other words, when performing a classical parametric test, the conclusion of the test could generally be invalidated if strictly speaking the data was not generated from the presumed class of parametric distributions. When the livelihood of a corporation is at stake in these choices, it might be wiser to avoid relying on such assumptions.

\subsection{Parameter Optimization: an example}

Consider the same problem of linear regression as described above but, let us now turn the task of parameter estimation. Our objective is to minimize the expected out-of-sample squared error which does not mean that we should minimize the in-sample mean squared error:

$$
M S E=\frac{1}{N} \sum_{i=1}^{N}\left(y_{i}-\left(\hat{\alpha}+\hat{\beta} x_{i}\right)\right)^{2}
$$

\footnotetext{
${ }^{1}$ the only assumption, in ordinary tests, is that the data points are generated i.i.d., independently from the same distribution. Even this assumption can be relaxed in order to deal with sequentially dependent data, e.g. as in (Newey \& West 1987, Diebold \& Mariano 1995, Campbell, Lo \& MacKinlay 1997, Chapados \& Bengio 2003).
} 
Minimizing the MSE is what the maximum likelihood calls for in the classical framework. The reason for that apparent discrepancy has to do with the statistical learning principles defined above. Instead, in order to obtain better generalization, we turn to the regularization framework and accordingly choose to minimize a penalized criterion leading to what is often referred to as ridge regression:

$$
M S E_{\lambda}=\frac{1}{N} \sum_{i=1}^{N}\left(y_{i}-\left(\hat{\alpha}+\hat{\beta} x_{i}\right)\right)^{2}+\lambda \hat{\beta}^{2}
$$

with $\lambda \geq 0$ and a minimum achieved at $\hat{\beta}_{\lambda}$. Thus $\hat{\beta}_{0}$ is the Ordinary Least Squares estimator. This minimum is always achieved with shrinked solutions, i.e. $\left\|\hat{\beta}_{\lambda}\right\|<\left\|\hat{\beta}_{0}\right\|$ for $\lambda>0$. Note that this solution is generally biased, unlike $\hat{\beta}_{0}$, in the sense that if the data is generated from a multivariate normal distribution, the expected value of $\left\|\hat{\beta}_{\lambda}\right\|$ is smaller than the true value $\|\beta\|$ from the underlying distribution.

In the case where linear regression is the proper model it is easy to show that the optimal fixed value of $\lambda$ is

$$
\lambda^{*}=\frac{\sigma^{2}}{N\|\beta\|^{2}} .
$$

Note therefore that the optimal model is biased (optimal in the sense of minimizing out-of-sample error). Considering the case of automobile insurance, with noisy observations (large $\sigma^{2}$ ) and the small sample effect (small $N$, as argued above), we obtain large optimal values for $\lambda$. In other words, for this particular case, regularization significantly differs from maximum likelihood.

This example illustrates the more general principle of bias-variance trade-off in generalization error, well discussed by (Geman, Bienenstock \& Doursat 1992). Increasing $\lambda$ corresponds to "smoothing more" in non-parametric statistics (choosing a simpler function) or to the choice of a smaller capacity ("smaller" class of functions) in Vapnik's 
VC-theory (Vapnik 1998). A too large value of $\lambda$ corresponds to underfitting (too simple model, too much bias), whereas a too small value corresponds to overfitting (too complex model, too much variance). Which value of $\lambda$ should be chosen? (the above formula is not practical because it requires the true $\beta$, and is only applicable if the data is really Gaussian). It should be the one that strikes the optimal balance between bias and variance. This is the question that model selection algorithms address. Fortunately, the expected outof-sample error has a unique minimum as a function of $\lambda$ (or more generally of the capacity, or complexity of the class of functions). Concerning the above formula, note that unfortunately the data is generally not normal, and $\sigma^{2}$ and $\beta$ are both unknown, so the above formula can't be used directly to choose $\lambda$. However, using a separate held-out data set (also called a validation set, here), and taking advantage of that unique minimum property (which is true for any data distribution), we can quickly select a good value of $\lambda$ (essentially by searching), which approximately minimizes the estimated out-of-sample error on that validation set.

Note that we arrive at the conclusion that a biased model is preferable because we set as our goal to minimize out-of-sample error. If our goal was to discover the underlying "truth", and if we could make very strong assumptions about the true nature of the data distribution, then the more classical statistical approach based on minimum variance unbiased estimators would be more appropriate. However, in the context of practical insurance premia estimation, we don't really know the form of the true data distribution, and we really care about how the model is going to perform in the future (at least for ratemaking).

\section{Mathematical Objectives}

The first goal of insurance premium modeling is to estimate the $e x$ pected claim amount for a given insurance contract for a future period 
(usually one-year). Here we consider that the amount is 0 when no claim is filed. Let $X \in \mathbf{R}^{n}$ denote the customer and contract input profile, a vector representing all the information known about the customer and the proposed insurance policy before the beginning of the contract. Let $A \in \mathbf{R}^{+}$denote the amount that the customer claims during the contract period; we shall assume that $A$ is non-negative. Our objective is to estimate this claim amount, which is the pure premium $p_{\text {pure }}$ of a given contract $x:^{2}$

$$
p_{\text {pure }}(x)=E_{A}[A \mid X=x] .
$$

where $E_{A}[\cdot]$ denotes expectation, i.e. the average over an infinite population, and $E_{A}[A \mid X=x]$ is a conditional expectation, i.e. the average over a subset of an infinite population, comprising only the cases satisfying the condition $X=x$.

\subsection{The Precision Criterion}

In practice, of course, we have no direct access to the quantity (5), which we must estimate. One possible criterion is to seek the most precise predictor, which minimizes the expected squared error (ESE) over the unknown distribution:

$$
E_{A, X}\left[(p(X)-A)^{2}\right],
$$

where $p(X)$ is a pure premium predictor and the expectation is taken over the random variables $X$ (input profile) and $A$ (total claim amount). Since $P(A, X)$, the true joint distribution of $A$ and $X$, is unknown, we can unbiasedly estimate the ESE performance of an estimator $p$ on a data set $D_{\text {test }}=\left\{\left\langle x_{i}, a_{i}\right\rangle\right\}_{i=1}^{N}$, as long as this data set is not used to choose $p$. We do so by using the mean squared error on that data set:

$$
\frac{1}{N} \sum_{\left\langle x_{i}, a_{i}\right\rangle \in D_{\text {test }}}\left(p\left(x_{i} ; \theta\right)-a_{i}\right)^{2},
$$

\footnotetext{
${ }^{2}$ The pure premium is distinguished from the premium actually charged to the customer, which must account for the underwriting costs (marketing, commissions, premium tax), administrative overhead, risk and profit loadings and other costs.
} 
where $\theta$ is the vector of parameters of the model used to compute the premiums. The vector $x_{i}$ represents the $i^{\text {th }}$ input profile of dataset $D_{\text {test }}$ and $a_{i}$ is the claim amount associated to that input profile. Thus, $D_{\text {test }}$ is a set of $N$ insurance policies. For each policy, $D_{\text {test }}$ holds the input profile and associated incurred amount. We will call the data set $D_{\text {test }}$ a test set. It is used only to independently assess the performance of a predictor $p$. To choose $p$ from a (usually infinite) set of possible predictors, one uses an estimator $L$, which obtains a predictor $p$ from a given training set $D$. Such an estimator is really a statistical learning algorithm (Hastie, Tibshirani \& Friedman 2001), yielding a predictor $p=L_{D}$ for a given data set $D$. What we call the squared bias of such an estimator is $E_{X}\left[\left(E_{A}[A \mid X]-E_{D}\left[L_{D}(X)\right]\right)^{2}\right]$, where $E_{D}\left[L_{D}(X)\right]$ is the average predictor obtained by considering all possible training sets $D$ (sampled from $P(A, X))$. It represents how far the average estimated predictor deviates from the ideal pure premium. What we call the variance of such an estimator is $E_{X, D}\left[\left(L_{D}(X)-E\left[L_{D}(X)\right]\right)^{2}\right]$. It represents how the particular predictor obtained with some data set $D$ deviates from the average of predictors over all data sets, i.e. it represents the sensitivity of the estimator to the variations in the training data and is related to the classical measure of credibility.

Is the mean squared error (MSE) on a test set an appropriate criterion to evaluate the predictive power of a predictor $p$ ? First one should note that if $p_{1}$ and $p_{2}$ are two predictors of $E_{A}[A \mid X]$, then the MSE criterion is a good indication of how close they are to $E_{A}[A \mid X]$, since by the law of iterated expectations,

$$
\begin{array}{r}
E_{A, X}\left[\left(p_{1}(X)-A\right)^{2}\right]-E_{A, X}\left[\left(p_{2}(X)-A\right)^{2}\right]= \\
E_{X}\left[\left(p_{1}(X)-E_{A}[A \mid X]\right)^{2}\right] E_{X}\left[\left(p_{2}(X)-E_{A}[A \mid X]\right)^{2}\right],
\end{array}
$$

and of course the expected MSE is minimized when $p(X)=E_{A}[A \mid X]$.

For the more mathematically-minded readers, we show the wellknown result that minimizing the expected squared error optimizes simultaneously both the precision (low bias) and the variance of the 
estimator. The expected squared error of an estimator $L_{D}$ decomposes as follows:

$$
\begin{aligned}
& E_{A, X, D}\left[\left(A-L_{D}(X)\right)^{2}\right] \\
= & E_{A, X, D}\left[\left(\left(A-E_{A}[A \mid X]\right)+\left(E_{A}[A \mid X]-L_{D}(X)\right)\right)^{2}\right] \\
= & \underbrace{E_{A, X}\left[\left(A-E_{A}[A \mid X]\right)^{2}\right]}_{\text {noise }}+E_{X, D}\left[\left(E_{A}[A \mid X]-L_{D}(X)\right)^{2}\right] \\
& +\underbrace{2 E_{A, X, D}\left[\left(A-E_{A}[A \mid X]\right)\left(E_{A}[A \mid X]-L_{D}(X)\right)\right]}_{\text {zero }} \\
= & E_{X, D}\left[\left(\left(E_{A}[A \mid X]-E_{D}\left[L_{D}(X)\right]\right)+\left(E_{D}\left[L_{D}(X)\right]-L_{D}(X)\right)\right)^{2}\right] \\
& + \text { noise } \\
= & +E_{X}\left[\left(E_{A}[A \mid X]-E_{D}\left[L_{D}(X)\right]\right)^{2}\right] \\
& +E_{X, D}\left[\left(E_{D}\left[L_{D}(X)\right]-L_{D}(X)\right)^{2}\right] \\
& +\underbrace{2 E_{X, D}\left[\left(E[A \mid X]-E_{D}\left[L_{D}(X)\right]\right)\left(E_{D}\left[L_{D}(X)\right]-L_{D}(X)\right)\right]}_{\text {zero }} \\
& + \text { noise } \\
= & \underbrace{E_{X}\left[\left(E_{A}[A \mid X]-E_{D}\left[L_{D}(X)\right]\right)^{2}\right]}_{\text {varian }^{2}} \\
& +\underbrace{E_{X, D}\left[\left(E_{D}\left[L_{D}(X)\right]-L_{D}(X)\right)^{2}\right]}_{\text {noise }} . \\
& + \text { noise }
\end{aligned}
$$

Thus, algorithms that try to minimize the expected squared error simultaneously reduce both the bias and the variance of the estimators, striking a tradeoff that minimizes the sum of both (since the remainder is the noise, which cannot be reduced by the choice of predictor). On the other hand, with a rule such as minimum bias used with table based methods, cells are merged up to a point where each cell has sufficient credibility, i.e., where the variance is sufficiently low. Then, once the credibility (and variance) level is set fixed, the bias is minimized. On the contrary, by directly targeting minimization of the expected squared error one avoids this arbitrary setting of a credibility level. 
As discussed in the previous section, in comparison to parametric approaches, this approach avoids distributional assumptions. Furthermore, it looks for an optimal trade-off between bias and variance, whereas parametric approaches typically focus on the unbiased estimators (within a class that is associated with a certain variance). Because of the above trade-off possibility, it is always possible (with a finite data set) to improve an unbiased estimator by trading a bit of bias increase for a lot of variance reduction.

\subsection{The Fairness Criterion}

In insurance policy pricing, the precision criterion is not the sole part of the picture; just as important is that the estimated premiums do not systematically discriminate against specific segments of the population. We call this objective the fairness criterion, sometimes referred to as actuarial fairness. We define the bias of the premium $b(P)$ to be the difference between the average pure premium and the average incurred amount, in a given sub-population $P$ of dataset $D$ :

$$
b(P)=\frac{1}{|P|} \sum_{\left\langle x_{i}, a_{i}\right\rangle \in P} p\left(x_{i}\right)-a_{i},
$$

where $|P|$ denotes the cardinality of the sub-population $P$, and $p(\cdot)$ is some premium estimation function. The vector $x_{i}$ represents the $i^{\text {th }}$ input profile of sub-population $P$ and $a_{i}$ is the claim amount associated to that input profile. A possible fairness criterion would be based on minimizing the sum, over a certain set of critical subpopulations $\left\{P_{k}\right\}$ of dataset $D$, of the square of the biases:

$$
\sum_{k, P_{k} \in D} b^{2}\left(P_{k}\right)
$$

In the particular case where one considers all sub-populations, then both the fairness and precision criterions lead to the same optimal solution, i.e., they are minimized when $p\left(x_{i}\right)=E\left[A_{i} \mid x_{i}\right], \forall i$, i.e., for every insurance policy, the premium is equal to the conditional expectation of the claim amount. The proof is given in appendix A. 
In spite of the above result, it might be interesting to measure fairness of particular sub-populations of interest. Business-wise, for example, it is important to know whether populations that pay low premium are overpaying (considering what they cost in claims) with respect to populations that pay a high premium. For this purpose, we used the following methodology: after training a model to minimize the MSE criterion (7), we define a finite number of disjoint subsets (subpopulations) of test set $D: P_{k} \subset D, P_{k} \cap P_{j \neq k}=\emptyset$, and verify that the absolute bias is not significantly different from zero. The subsets $P_{k}$ can be chosen at convenience; in our experiments, we considered 10 subsets of equal size delimited by the deciles of the test set premium distribution. In this way, we verify that, for example, for the group of contracts with a premium between the 5th and the 6th decile, the average premium matches, within statistical significance, the average claim amount.

\section{$4 \quad$ Methodology}

A delicate problem to guard against when applying statistical learning algorithms is that of overfitting. It has precisely to do with striking the right trade-off between bias and variance (as introduced earlier), and is known in technical terms as capacity control. Figure 1 illustrates the problem: the two plots show empirical data points (black dots) that we are trying to approximate with a function (solid red curve). All points are sampled from the same underlying function (dashed blue curve), but are corrupted with noise; the dashed curve may be seen as the "true" function we are seeking to estimate.

The left plot shows the result of fitting a very flexible function, i.e. a high-order polynomial in this case, to the available data points. We see that the function fits the data points perfectly: there is zero error (distance) between the red curve and each of the black dots. However, the function oscillates wildly between those points; it has not captured any of the fundamental features of the underlying function. What is 
happening here is that the function has mostly captured the noise in the data: it overfits.

The right plot, on the other hand, shows the fitting of a less flexible function, i.e. a 2nd-order polynomial, which exhibits a small error with respect to each data point. However, by not fitting the noise (because it does not have the necessary degrees of freedom), the fitted function far better conveys the structural essence of the matter.

Capacity control lies at the heart of a sound methodology for data mining and statistical learning algorithms. The goal is simple: to choose a function class flexible enough (with enough capacity) to express a desired solution, but constrained enough that it does not fit the noise in the data points. In other words, we want to avoid overfitting and underfitting.

Figure 2 illustrates the basic steps that are commonly taken to resolve this issue: these are not the only means to prevent overfitting, but are the simplest to understand and use.

1. The full data set is randomly split into three disjoint subsets, respectively called the training, validation, and test sets.
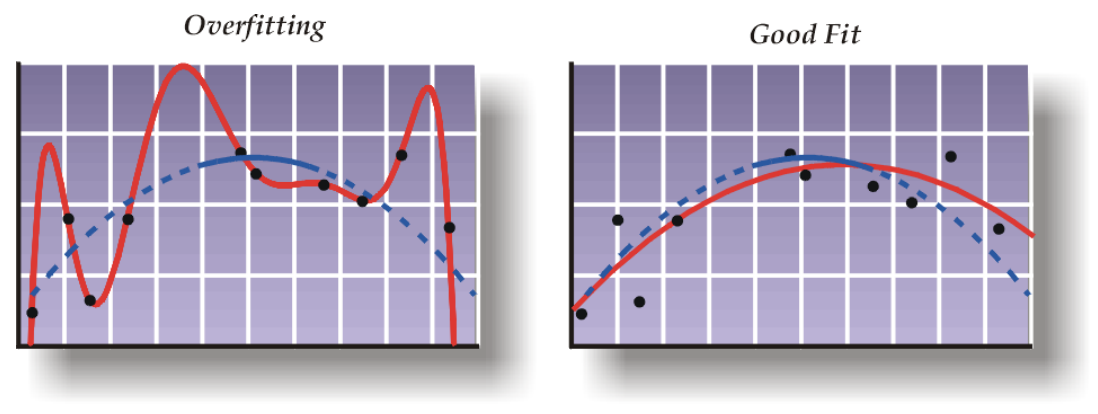

Figure 1. Illustration of overfitting. The solid left curve fits the noise in the data points (black dots) and has not learned the underlying structure (dashed). The right curve, with less flexibility, does not overfit. 


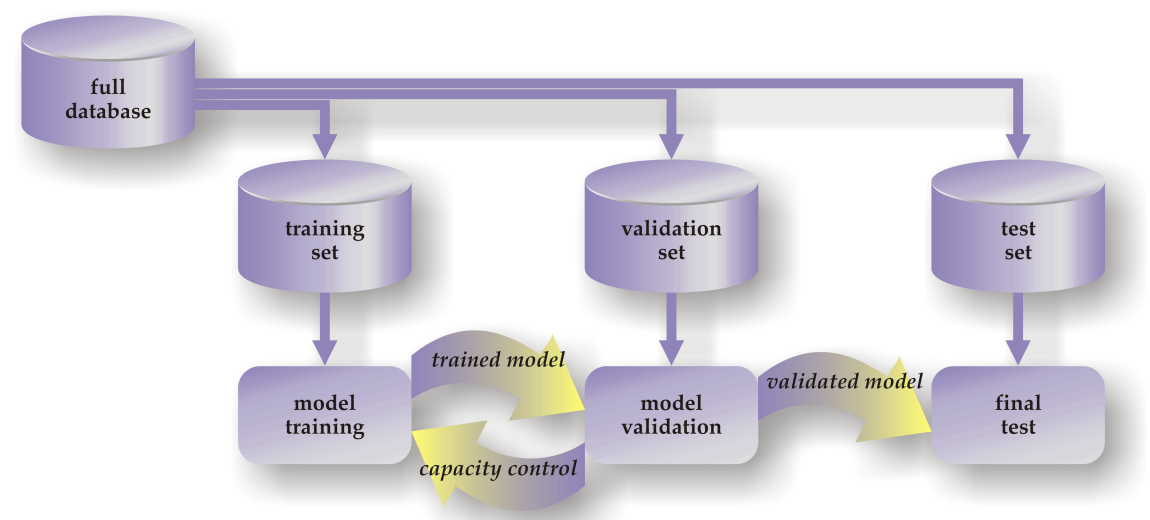

Figure 2. Methodology to prevent overfitting. Model capacity is controlled via a validation set, disjoint from the training set. The generalization performance estimator is obtained by final testing on the test set, disjoint from the first two.

2. The training set is used to fit a model with a chosen initial capacity.

3. The validation set is used to evaluate the performance of that fitted function, on different data points than used for the fitting, i.e. cases not in the training set. The key here is that a function overfitting the training set will exhibit a poor performance on the validation set, if it does not capture the underlying structure of the problem.

4. Depending on the validation set performance, the capacity of the model is adjusted (increased or reduced), and a new training phase (step 2) is attempted. This training-validation cycle is repeated multiple times and the capacity that provides the best validation performance (among those tried) is retained. This is really an optimization of the capacity and can formally be handled by numerical optimization algorithms.

5. Finally, the performance of the "ultimate" function (that coming out of the validation phase) is evaluated on data points never used 
previously - those in the test set - to give a completely unbiased measure of the performance that can be expected when the system is deployed in the field. This is called generalization performance.

In the case of automobile insurance, there is a sequential structure that must be respected. When using data from previous years to simulate the out-of-sample performance of models, one should try to replicate as closely as possible the actual process that will be followed to deploy a model. A sound procedure is to split the data according to their policy date year into the training (year $y-3$ ), validation (year $y-2$ ) and test (year $y$ ) sets. The reason for skipping year $y-1$ is to recognize the fact that at time $y$, as the model is deployed, year $y-1$ is not yet available. Reducing this gap to a few months would help the insurer account for more recent data and very likely obtain better performance. An insurer having access to data dating from 1995 could obtain test performances for years 1998 and above. Assuming 2002 results are available leads to 5 estimates of the test performance of the whole modelling procedure.

\section{$5 \quad$ Models}

In this section, we describe various models that have been implemented and used for the purpose of ratemaking, and evaluated in this study. We begin with the simplest model: charging a flat premium to every insured. Then, we gradually move on towards more complex models.

\subsection{Constant Model}

For benchmark evaluation purposes, we implemented the constant model. This consists of simply charging every single insurance policy a flat premium, regardless of the associated variable values. The premium is the mean of all incurred amounts as it is the constant value 
that minimizes the mean-squared error.

$$
p(x)=\beta_{0},
$$

where $\beta_{0}$ is the mean and the premium $p(x)$ is independent of the input profile $x$. In figure 3 , the constant model is viewed as a flat line when the premium value is plotted against one of the input variables.

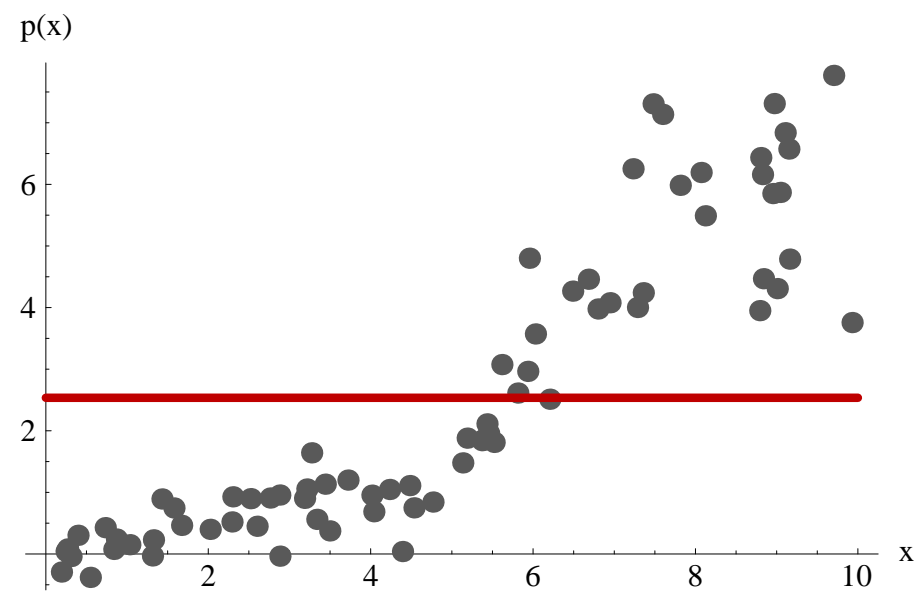

Figure 3. The constant model fits the best horizontal line through the training data.

\subsection{Linear Model}

We implemented a linear model which consists of a set of coefficients, one for each variable plus an intercept value, that minimize the meansquared error,

$$
p(x)=\beta_{0}+\sum_{i=1}^{n} \beta_{i} x_{i} .
$$

Figure 4 illustrates a linear model where the resulting premiums form a line, given one input variable value. With a two dimensional input 


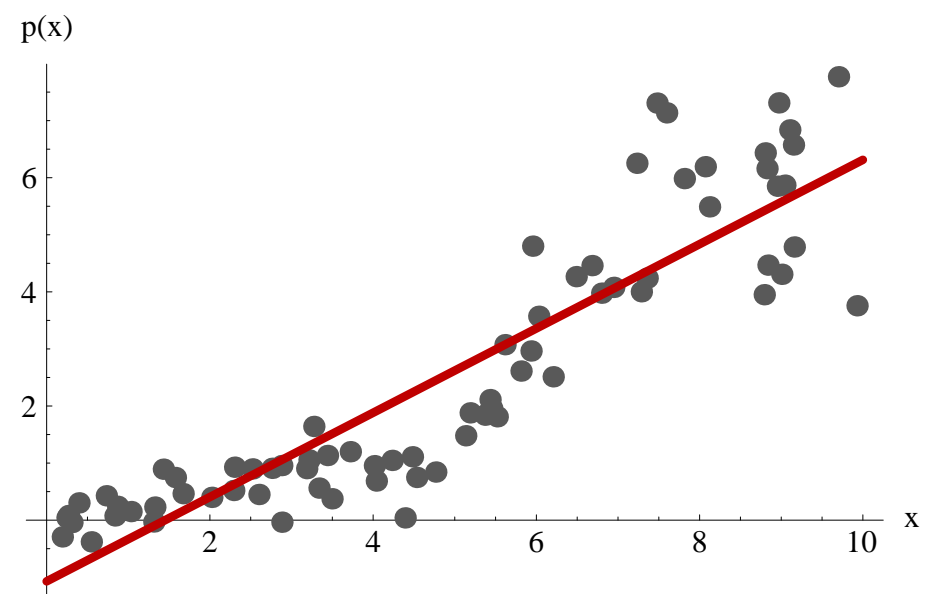

Figure 4. The linear model fits a straight line through the training data.

variable space, a plane would be drawn. In higher dimension, the corresponding geometrical form is referred to as a hyper-plane.

There are two main ways to control the capacity of linear models when in presence of noisy data:

- using a subset of input variables; this directly reduces the number of coefficients (but choosing the best subset introduces another level of choice which is sometimes detrimental).

- penalizing the norm of the parameters (in general excluding the intercept $\beta_{0}$ ); this is called ridge regression in statistics, and weight decay in the Neural Networks community. This was the main method used to control capacity of the linear model in our experiments (see above subsection 2.2).

It should be noted that the premium computed with the linear model can be negative (and negative values are indeed sometimes obtained with the trained linear models). This may happen even if there are no negative amounts in the data, simply because the model has no 
built-in positivity constraint (unlike the GLM and the softplus Neural Network described below).

\subsection{Table-based methods}

These more traditional ratemaking methods rely mainly on a classification system, base rates and relativities. The target function is approximated by constants over regular (finite) intervals. As shown on the figure, this gives rise to a typical staircase-like function, where each level of the staircase is given by the value in the corresponding cell in the table. A common refinement in one dimension is to perform a linear interpolation between neighboring cells, to smooth the resulting function somewhat. The table is not limited to two variables; however, when adding a new variable (dimension), the number of cells increases by a factor equal to the number of discretization steps in the new variable.

In order to use table-based methods to estimate a pure premium, find a certain number of variables deemed useful for the prediction, and discretize those variables if they are continuous. To fill out the table, compute over a number of years (using historical data) the total incurred claim amount for all customers whose profiles fall within a given cell of the table, and average the total within that cell. This gives the pure premium associated with each cell of the table.

Assuming that the $i^{\text {th }}$ variable of profile $x$ belongs to the $j^{\text {th }}$ category, we obtain,

$$
p(x)=\beta_{0} \prod_{i=1}^{m} \beta_{i, j}+\sum_{i=m+1}^{n} \beta_{i, j},
$$

where $\beta_{i, j}$ is the relativity for the $j^{\text {th }}$ category of the $i^{\text {th }}$ variable and $\beta_{0}$ is the standard premium. We consider the case where the first $m$ factors are multiplicative and the last $n-m$ factors are additive.

The formula above assumes that all variables have been analyzed in- 
dividually and independently. A great deal of effort is often put in trying to capture dependencies (or interactions) between some variables and to encode them into the premium model.

An extension of the above is to multiplicatively combine multiple tables associated to different subsets of variables. This is in effect a particular form of Generalized Linear Model (see below), where each table represents the interdependence effects between some variables.

\subsection{Greedy Multiplicative Model}

Greedy learning algorithms "grow" a model by gradually adding one "piece" at a time to the model, but keeping the already chosen pieces fixed. At each step, the "piece" that is most helpful to minimize the training criterion is "added" to the model. This is how decision trees are typically built. Using the validation set performance we can decide when to stop adding pieces (when the estimated out-of-sample performance starts degrading).

The GLM described in the next section is a multiplicative model because the final premium function can be seen as a product of coefficients associated with each input variable. The basic idea of the Greedy Multiplicative Model is to add one of these multiplicative coefficients at a time. At each step, we have to choose one among the input variables. We choose the variable which would reduce most the training MSE. The coefficient for that component is easily obtained analytically by minimizing the MSE when all the previously obtained coefficients are kept fixed.

In the tables we use the short-hand name "CondMean" for this model because it estimates and combines many conditional means. Note that like the GLM, this model provides positive premiums.

\subsection{Generalized Linear Model}


(Bailey \& Simon 1960) introduced Generalized Linear Models (GLM) to the actuarial community. More recently, (Brown 1988, Holler, Sommer \& Trahair 1999, Murphy, Brockman \& Lee 2000) conducted experiments using such models. GLMs, at their roots, are simple linear models that are composed with a fixed nonlinearity (the so-called link function); a commonly-used link function is simply the exponential function $e^{x}$. GLMs (with the exponential link) are sometimes used in actuarial modeling since they naturally represent multiplicative effects, for example risk factors whose effects should combine multiplicatively rather than additively. They are attractive since they incorporate problem-specific knowledge directly into the model. These models can be used to obtain a pure premium, yielding such a formula,

$$
p(x)=\exp \left(\beta_{0}+\sum_{i=1}^{n} \beta_{i} x_{i}\right),
$$

where, the exponentiation ensures that the resulting premiums are all positive. In figure 5, we can see that the model generates an exponential function in terms of the input variable.

In their favor, GLMs are quite easy to estimate ${ }^{3}$, have interpretable parameters, can be associated to parametric noise models, and are not so affected when the number of explanatory variables increases, as long as the number of observations used in the estimation remains sufficient. Unfortunately, they are fairly restricted in the shape of the functions they can estimate.

The capacity of a GLM model can be controlled using the same techniques as those mentionned above (5.2) in the context of linear models. Again, note that the GLM always provides a positive premium.

\footnotetext{
${ }^{3}$ We have estimated the parameters to minimize the mean-squared error, but other training criteria have also been proposed in the GLM literature and this could be the subject of further studies.
} 


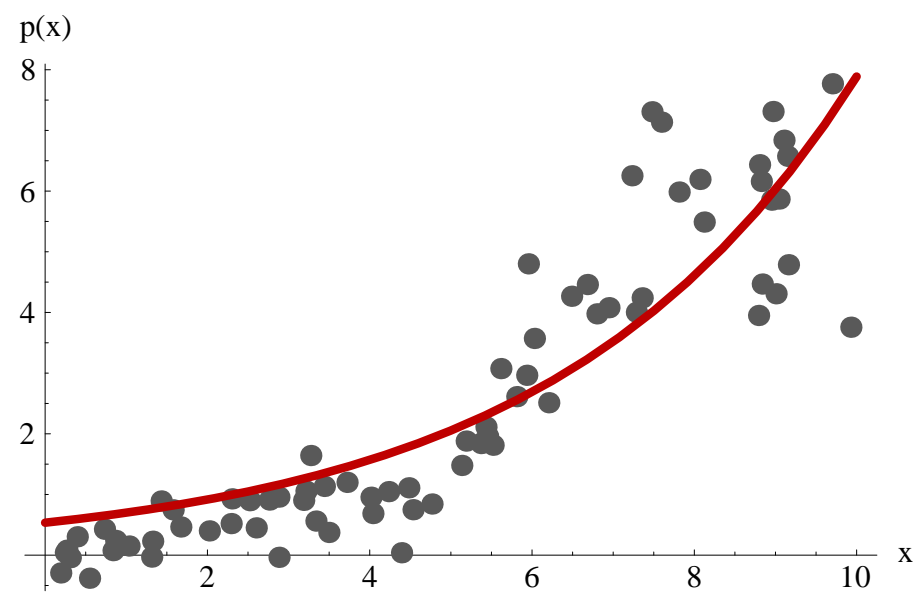

Figure 5. The Generalized Linear Model fits an exponential of a linear transformation of the variables.

\subsection{CHAID decision trees}

Decision trees split the variable space in smaller subspaces. Any input profile $x$ fits into one and only one of those subspaces called leaves. To each leaf is associated a different premium level,

$$
p(x)=\sum_{i=1}^{n_{l}} \mathbf{I}_{\left\{x \in l_{i}\right\}} \beta_{i}
$$

where $\mathbf{I}_{\left\{x \in l_{i}\right\}}$ is an indicator function equal to 1 if and only if $x$ belongs to the $i^{\text {th }}$ leaf. In that case, $\mathbf{I}_{\left\{x \in l_{i}\right\}}=1$ and $p(x)=\beta_{i}$. Otherwise, $\mathbf{I}_{\left\{x \in l_{i}\right\}}$ is equal to zero, meaning $x$ belongs to another leaf. The number of leaves is $n_{l}$. The premium level $\beta_{i}$ is set equal to the average incurred amount of the policies for which the profile $x$ belongs to the $i^{\text {th }}$ leaf. In figure 6 , the decision tree is viewed as generating a piecewise constant function. The task of the decision tree is to choose the "best" possible partition of the input variable space.

The basic way in which capacity is controlled is through several hyperparameters: minimum population in each leaf, minimum population 


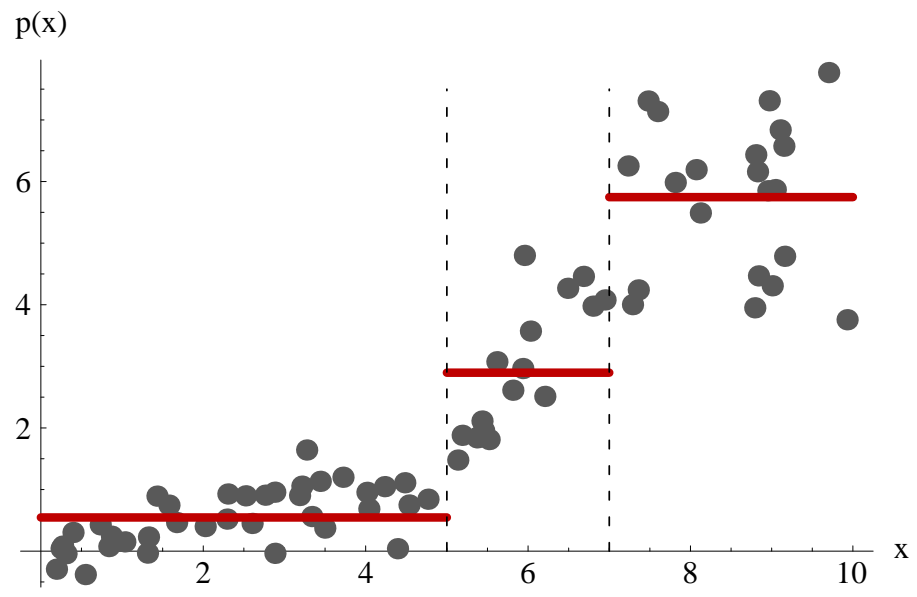

Figure 6. The CHAID model fits constants to partitions of the variables. The dashed lines in the figure delimit the partitions, and are found automatically by the CHAID algorithm.

to consider splitting a node, maximum height of the decision tree and Chi-square statistic threshold value.

\subsection{Combination of CHAID and Linear Model}

This model is similar to the previous one except that, in each leaf, we have replaced the associated constant premium value with a linear regression. Each leaf has its own set of regression coefficients. There are thus $n_{l}$ different linear regressions of $n+1$ coefficients each.

$$
p(x)=\sum_{i=1}^{n_{l}} \mathbf{I}_{\left\{x \in l_{i}\right\}}\left(\beta_{i, 0}+\sum_{j=1}^{n} \beta_{i, j} x_{j}\right) .
$$

Each linear regression was fit to minimize the mean-squared error on the training cases that belong to its leaf. For reasons that are clear in the light of learning theory, a tree used in such a combination should 
have less leaves than an ordinary CHAID tree. In our experiments we have chosen the size of the tree based on the validation set MSE.

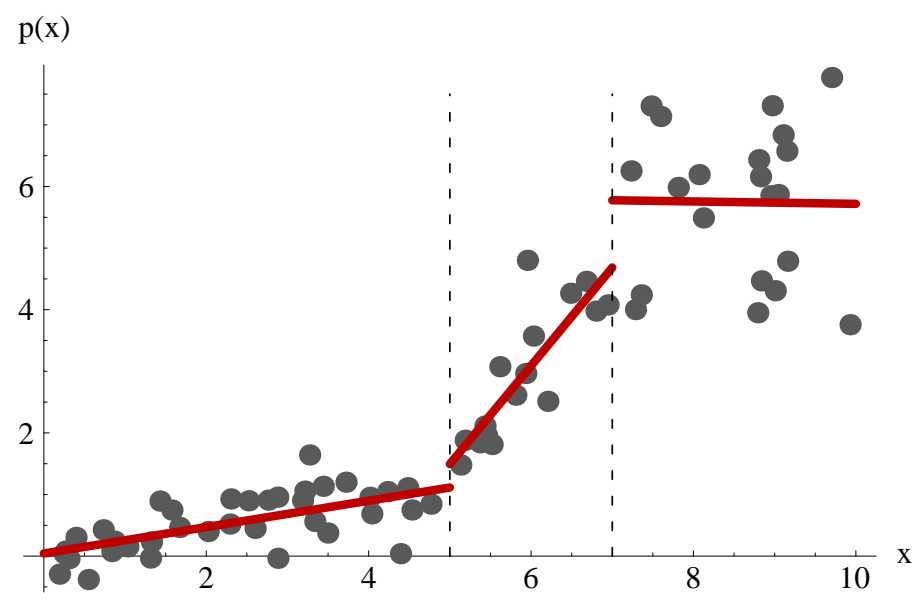

Figure 7. The CHAID+Linear model fits a straight line within each of the CHAID partitions of the variable space.

In these models, capacity is controlled with the same hyperparameters as CHAID, and there is also the question of finding the right weight decay for the linear regression. Again, the validation set is used for this purpose.

\subsection{Ordinary Neural Network}

Ordinary Neural Networks consist of the clever combination and simultaneous training of a group of units or neurons that are individually quite simple. Figure 8 illustrates a typical multi-layer feedforward architecture such as the ones that were used for the current project.

We describe here the steps that lead to the computation of the final output of the Neural Network. First, we compute a series of linear 


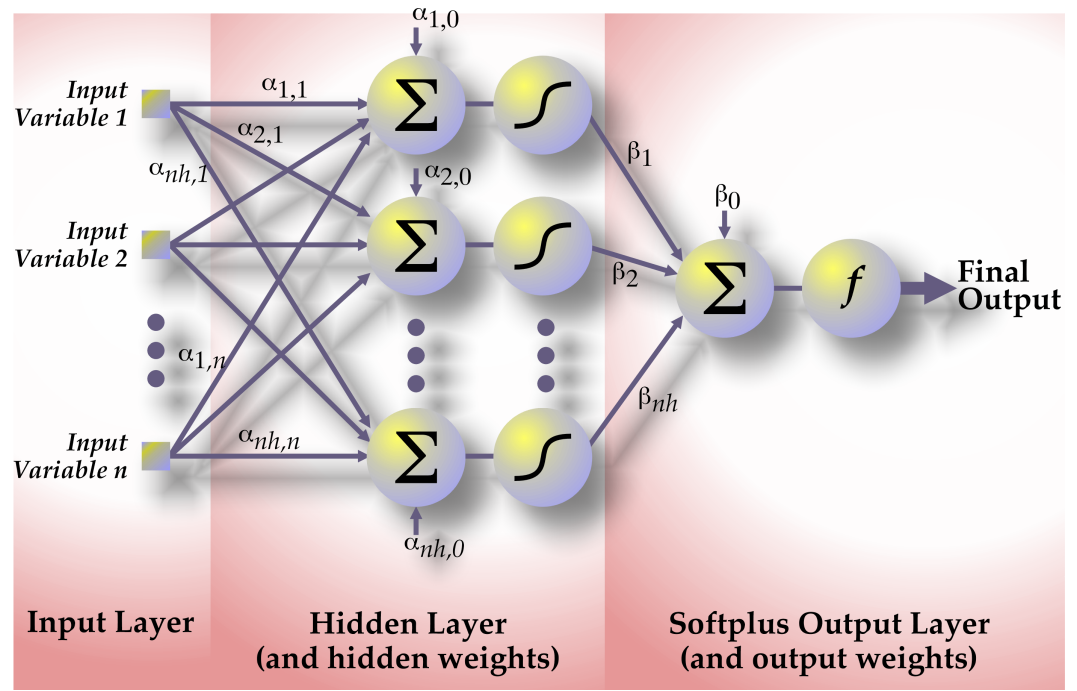

Figure 8. Topology of a one-hidden-layer Neural Network. In each unit of the hidden layer, the variables are linearly combined. The network then applies a non-linear transformation on those linear combinations. Finally, the resulting values of the hidden units are linearly combined in the output layer.

combinations of the input variables:

$$
v_{i}=\alpha_{i, 0}+\sum_{j=1}^{n} \alpha_{i, j} x_{j},
$$

where $x_{j}$ is the $j^{\text {th }}$ out of $n$ variables, $\alpha_{i, 0}$ and $\alpha_{i, j}$ are the slope intercept and the weights of the $i^{\text {th }}$ linear combination. The result of the linear combination, $v_{i}$, represents a projection in a preferred direction, that combines information from potentially all the input variables.

Then, a non-linear transform (called a transfer function) is applied to each of the linear combinations in order to obtain what are called 
the hidden units. We used the hyperbolic tangent function:

$$
\begin{aligned}
h_{i} & =\tanh \left(v_{i}\right) \\
& =\frac{e^{v_{i}}-e^{-v_{i}}}{e^{v_{i}}+e^{-v_{i}}},
\end{aligned}
$$

where $h_{i}$ is the $i^{\text {th }}$ hidden unit. The use of such a transfer function with infinite expansion in its terms has an important role in helping the Neural Network capture nonlinear interactions and this is the subject of subsection 5.9.

Finally, the hidden units are linearly combined in order to compute the final output of the Neural Network:

$$
p(x)=\beta_{0}+\sum_{i=1}^{n_{h}} \beta_{i} h_{i},
$$

where $p(x)$ is the premium computed by the Neural Network, $n_{h}$ is the number of hidden units and $\beta_{0}$ and $\beta_{i}$ are the slope intercept and the weights of the final linear combination.

Put all together in a single equation, we obtain:

$$
p(x)=\beta_{0}+\sum_{i=1}^{n_{h}} \beta_{i} \tanh \left(\alpha_{i, 0}+\sum_{j=1}^{n} \alpha_{i, j} x_{j}\right) .
$$

Figure 9 depicts a smooth non-linear function which could be generated by a Neural Network.

The number of hidden units ( $n_{h}$ above) plays a crucial role in our desire to control the capacity of the Neural Network. If we choose a value for $n_{h}$ that is too large, we obtain overfitting: the number of parameters of the model increases and it becomes possible, during the parameter optimization phase, for the Neural Network to model noise or spurious dependencies. These dependencies, present in the training dataset used for optimization, might not apply to other datasets. 


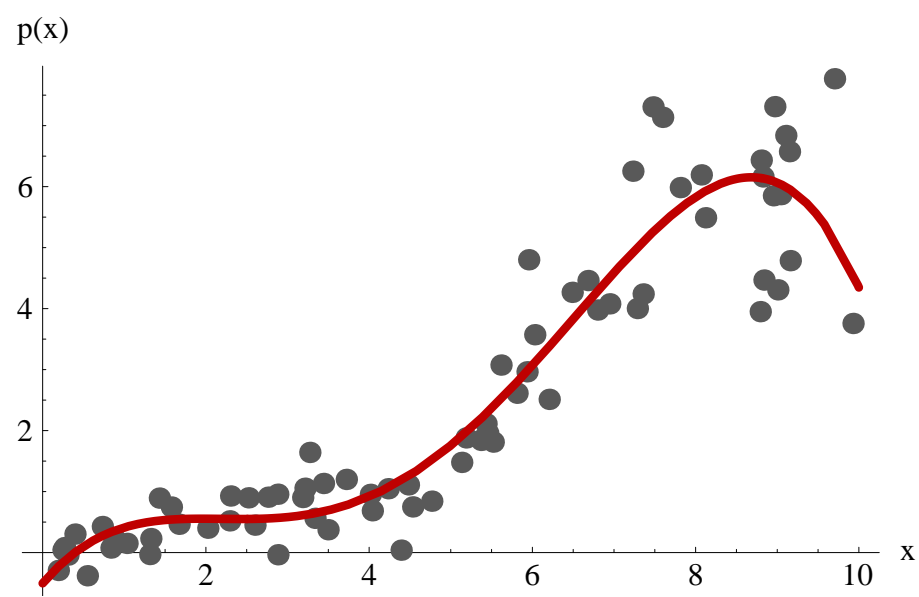

Figure 9. The Neural Network model learns a smooth non-linear function of the variables.

Conversely, setting $n_{h}$ to a value that is too low corresponds to underfitting: the number of parameters becomes too small and the Neural Network can not capture all of the relevant interactions in order to properly compute the premiums.

Thus, choosing the optimal number of hidden units is an important part of modeling with Neural Networks. Another technique for controlling the capacity of a Neural Network is to use weight decay, i.e., a penalized training criterion as described in subsection 2.2 that limits the size of the parameters of the Neural Network.

Choosing the optimal values for the parameters is a complex task and out of the scope of this chapter. Many different optimization algorithms and refinements have been suggested (Bishop 1995, Orr \& Müller 1998) but in practice, the simple stochastic gradient descent algorithm is still very popular and usually gives good performance.

Note that like the linear regression, this model can potentially yield negative premiums in some cases. We have observed much fewer such 
cases than with the linear regression.

\subsection{How can Neural Networks Represent Nonlinear Interactions?}

For the more mathematically-minded readers, we present a simple explanation of why Neural Networks are able to represent nonlinear interactions between the input variables. To simplify, suppose that we have only two input variables, $x_{1}$ and $x_{2}$. In classical linear regression, a common trick is to include fixed nonlinear combinations among the regressors, such as $x_{1}^{2}, x_{2}^{2}, x_{1} x_{2}, x_{1}^{2} x_{2}, \ldots$ However, it is obvious that this approach adds exponentially many terms to the regression, as one seeks higher powers of the input variables.

In contrast, consider a single hidden unit of a Neural Network, connected to two inputs. The adjustable network parameters are named, for simplicity, $\alpha_{0}, \alpha_{1}$ and $\alpha_{2}$. A typical function computed by this unit is given by

$$
\tanh \left(\alpha_{0}+\alpha_{1} x_{1}+\alpha_{2} x_{2}\right) \text {. }
$$

Here comes the central part of the argument: performing a Taylor series expansion of $\tanh \left(y+\alpha_{0}\right)$ in powers of $y$, and letting $\alpha_{1} x_{1}+\alpha_{2} x_{2}$ stand for $y$, we obtain (where $\beta \equiv \tanh \alpha_{0}$ ),

$$
\begin{aligned}
& \tanh \left(\alpha_{0}+\alpha_{1} x_{1}+\alpha_{2} x_{2}\right)= \\
& \quad \beta+\left(1-\beta^{2}\right)\left(\alpha_{1} x_{1}+\alpha_{2} x_{2}\right)+\left(-\beta+\beta^{3}\right)\left(\alpha_{1} x_{1}+\alpha_{2} x_{2}\right)^{2}+ \\
& \quad\left(-\frac{1}{3}+\frac{4 \beta^{2}}{3}-\beta^{4}\right)\left(\alpha_{1} x_{1}+\alpha_{2} x_{2}\right)^{3}+ \\
& \quad\left(\frac{2 \beta}{3}-\frac{5 \beta^{3}}{3}+\beta^{5}\right)\left(\alpha_{1} x_{1}+\alpha_{2} x_{2}\right)^{4}+O\left(\alpha_{1} x_{1}+\alpha_{2} x_{2}\right)^{5} .
\end{aligned}
$$

In fact the number of terms is infinite: the nonlinear function computed by this single hidden unit includes all powers of the input variables, but they cannot all be independently controlled. The terms that will ultimately stand out depend on the coefficients $\alpha_{0}, \alpha_{1}$, and $\alpha_{2}$. Adding more hidden units increases the flexibility of the overall function computed by the network: each unit is connected to the 
input variables with its own set of coefficients, thereby allowing the network to capture as many (nonlinear) relationships between the variables as the number of units allows.

The coeffients linking the input variables to the hidden units can also be interpreted in terms of projections of the input variables. Each set of coefficients for one unit represents a direction of interest in input space. The values of the coefficients are found during the network training phase using iterative nonlinear optimization algorithms.

\subsection{Softplus Neural Network}

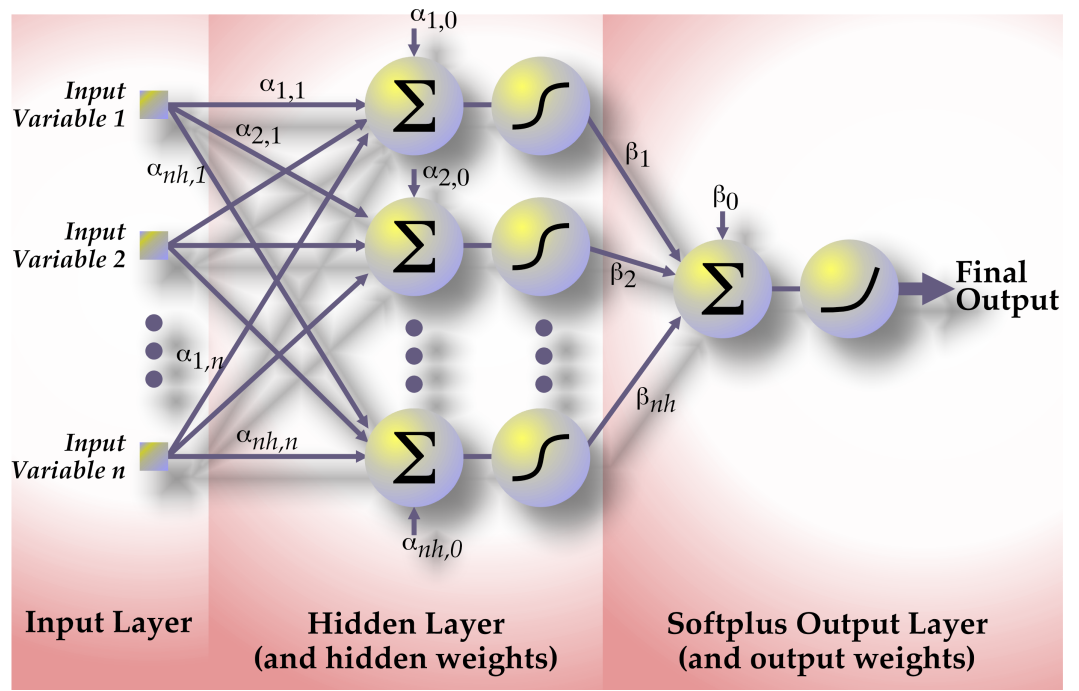

Figure 10. Topology of a one-hidden-layer softplus Neural Network. The hidden layer applies a non-linear transformation of the variables, whose results are linearly combined by the output layer. The softplus output function forces the function to be positive. To avoid cluttering, some weights linking the variables to the hidden layer are omitted on the figure.

This new type of model was introduced precisely to make sure that positive premiums are obtained. The softplus function was recently introduced in (Dugas, Bengio, Bélisle, Nadeau \& Garcia 2001) as a means to model a convex relationship between an output and one of 
its inputs. We modified the Neural Network architecture and included a softplus unit as a final transfer function. Figure 10 illustrates this new architecture we have introduced for the purpose of computing insurance premiums. The corresponding formula is as such:

$$
p(x)=F\left(\beta_{0}+\sum_{i=1}^{n_{h}} \beta_{i} \tanh \left(\alpha_{i, 0}+\sum_{j=1}^{n} \alpha_{i, j} x_{j}\right)\right),
$$

where $F(\cdot)$ is the softplus function which is actually simply the primitive (integral) function of the "sigmoid" function. Thus

$$
F(y)=\log \left(1+e^{y}\right) .
$$

The softplus function is convex and monotone increasing w.r.t. to its input and always strictly positive. Thus, as can be seen in Figure 11, this proposed architecture leads to strictly positive premiums.

In preliminary experiments we have also tried to use the exponential function (rather than the softplus function) as the final transfer function. However we obtained poor results due to difficulties in the optimization (probably due to the very large gradients obtained when the argument of the exponential is large).

The capacity of the softplus Neural Network is tuned just like that of an ordinary Neural Network. Note that this kind of Neural Network architecture is not available in commercial Neural Network packages.

\subsection{Regression Support Vector Machine}

Support Vector Machines (SVM) have recently been introduced as a very powerful set of non-parametric statistical learning algorithms (see (Vapnik 1998) and (Schölkopf, Burges \& Smola 1998)). They have been very successful in classification tasks, but the framework has also been extended to perform regression. Like other kernel methods the class of functions has the following form:

$$
p(x)=\sum_{i} \alpha_{i} K\left(x, x_{i}\right)
$$




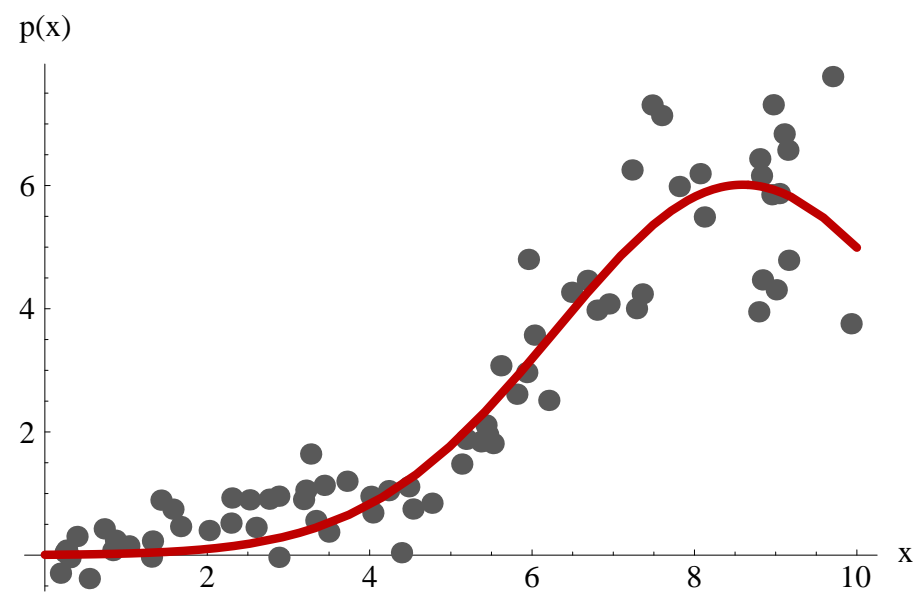

Figure 11. The softplus Neural Network model learns a smooth non-linear positive function of the variables. This positivity is desirable for estimating insurance premiums.

where $x_{i}$ is the input profile associated with one of the training records, and $\alpha_{i}$ is a scalar coefficient that is learned by the algorithm and $K$ is a kernel function that satisfies the Mercer condition (Cristianini \& Shawe-Taylor 2000):

$$
\int_{\mathcal{C}} K(x, y) g(x) g(y) d x d y \geq 0
$$

for any square integrable function $g(x)$ and compact subset $\mathcal{C}$ of $\mathbf{R}^{n}$. This Mercer condition ensures that the kernel function can be represented as a simple dot product:

$$
K(x, y)=\phi(x) \cdot \phi(y)
$$

where $\phi()$ is a function that projects the input profile vector into a (usually very) high-dimensional "feature" space, usually in a nonlinear fashion. This leads us, to a simple expression for the premium 
function:

$$
\begin{aligned}
p(x) & =\sum_{i} \alpha_{i} \phi(x) \cdot \phi\left(x_{i}\right) \\
& =\phi(x) \cdot\left(\sum_{i} \alpha_{i} \phi\left(x_{i}\right)\right) \\
& =w \cdot \phi(x) .
\end{aligned}
$$

Thus, in order to compute the premium, one needs to project input profile $x$ in its feature space and compute a dot product with vector $w$. This vector $w$ depends only on a certain number of input profiles from the training dataset and their associated coefficients. These input profiles are referred to as the support vectors and have been selected, along with their associated coefficients by the optimization algorithm.

SVMs have several very attractive theoretical properties, including the fact that an exact solution to the optimization problem of minimizing the training criterion can be found, and the capacity of the model is automatically determined from the training data. In many applications, we also find that most of the $\alpha_{i}$ coefficients are zero.

However, in the case of insurance data, an important characteristic of regression SVMs is that they are NOT trained to minimize the training MSE. Instead they minimize the following criterion:

$$
J=\frac{1}{2}\|w\|^{2}+\lambda \sum_{i}\left|a_{t}-p\left(x_{i}\right)\right|_{\epsilon}
$$

where $|e|_{\epsilon}=\max (0,|e|-\epsilon), \lambda$ and $\epsilon$ trade-off accuracy with complexity, $a_{i}$ is the observed incurred claim amount for record $i, x_{i}$ is the input profile for record $i$, and the vector $w$ is defined in terms of the $\alpha_{i}$ coefficients above. It can therefore be seen that this algorithm minimizes something close to the absolute value of the error rather than the squared error. As a consequence, the SVM tends to find a solution that is close to the conditional median rather than the conditional expectation, the latter being what we want to evaluate in 
order to set the proper value for a premium. Furthermore, note that the insurance data display a highly asymmetric distribution, so the median and the mean are very different. In fact, the conditional median is often exactly zero. Capacity is controlled through the $\epsilon$ and $\lambda$ coefficients.

\subsection{Mixture Models}

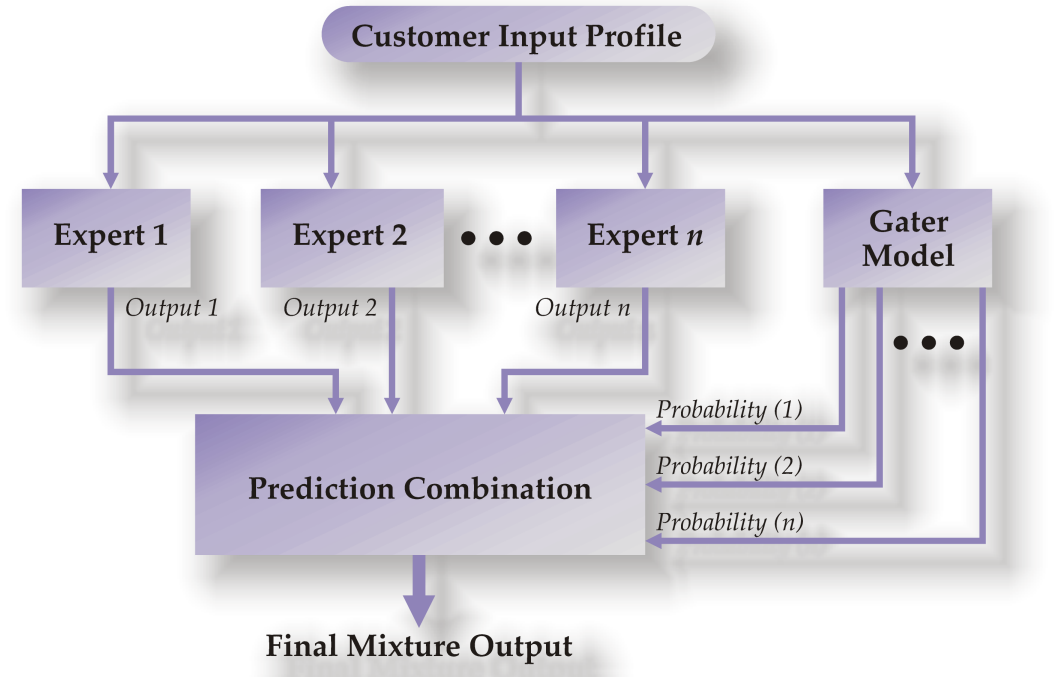

Figure 12. Schematic representation of the mixture model. The first-stage models each make an independent decision, which are linearly combined by a second-stage gater.

The mixture of experts has been proposed (Jacobs, Jordan, Nowlan \& Hinton 1991) in the statistical learning litterature in order to decompose the learning problem, and it can be applied to regression as well as classification. The conditional expectation is expressed as a linear combination of the predictions of expert models, with weights determined by a gater model. The experts are specialized predictors that each estimate the pure premium for insureds that belong to a certain class. The gater attempts to predict to which class each 
insured belongs, with an estimator of the conditional probability of the class given the insured's input profile. For a mixture model, the premium can be expressed as

$$
p(x)=\sum_{c} p(c \mid x) p_{c}(x)
$$

where $p(c \mid x)$ is the probability that an insured with input profile $x$ belongs to class $c$. This value is determined by the gater model. Also, $p_{c}(x)$ is the premium, as computed by the expert model of class $c$, associated to input profile $x$.

A trivial case occurs when the class $c$ is deterministically found for any particular input profile $x$. In that case, we simply split the training database and train each expert model on a subset of the data. The gater then simply assigns a value of $p_{c}(x)=1$ if $c$ is the appropriate class for input profile $x$ and zero otherwise. This is in fact fundamentally equivalent to other techniques such as decision trees or table-based methods. A more general and powerful approach is to have the learning algorithm discover a relevant decomposition of the data into different regions of the input space which then become the classes and are encoded in the gater model. In that case, both the gater and the experts are trained together.

In this study both the experts and the gater are softplus Neural Networks, but any other model can be used. In Figure 12, we schematically illustrate a mixture model as the one that was used in the framework of this project.

\section{Experimental Results}

\subsection{Mean-Squared Error Comparisons}

Table 1 summarizes the main results concerning the comparison between different types of statistical machine learning algorithms. All 
Table 1. Comparison between the main models, with MSE on the training set, validation set, and test sets. The MSE is with respect to claim amounts and premiums expressed in thousand of dollars.

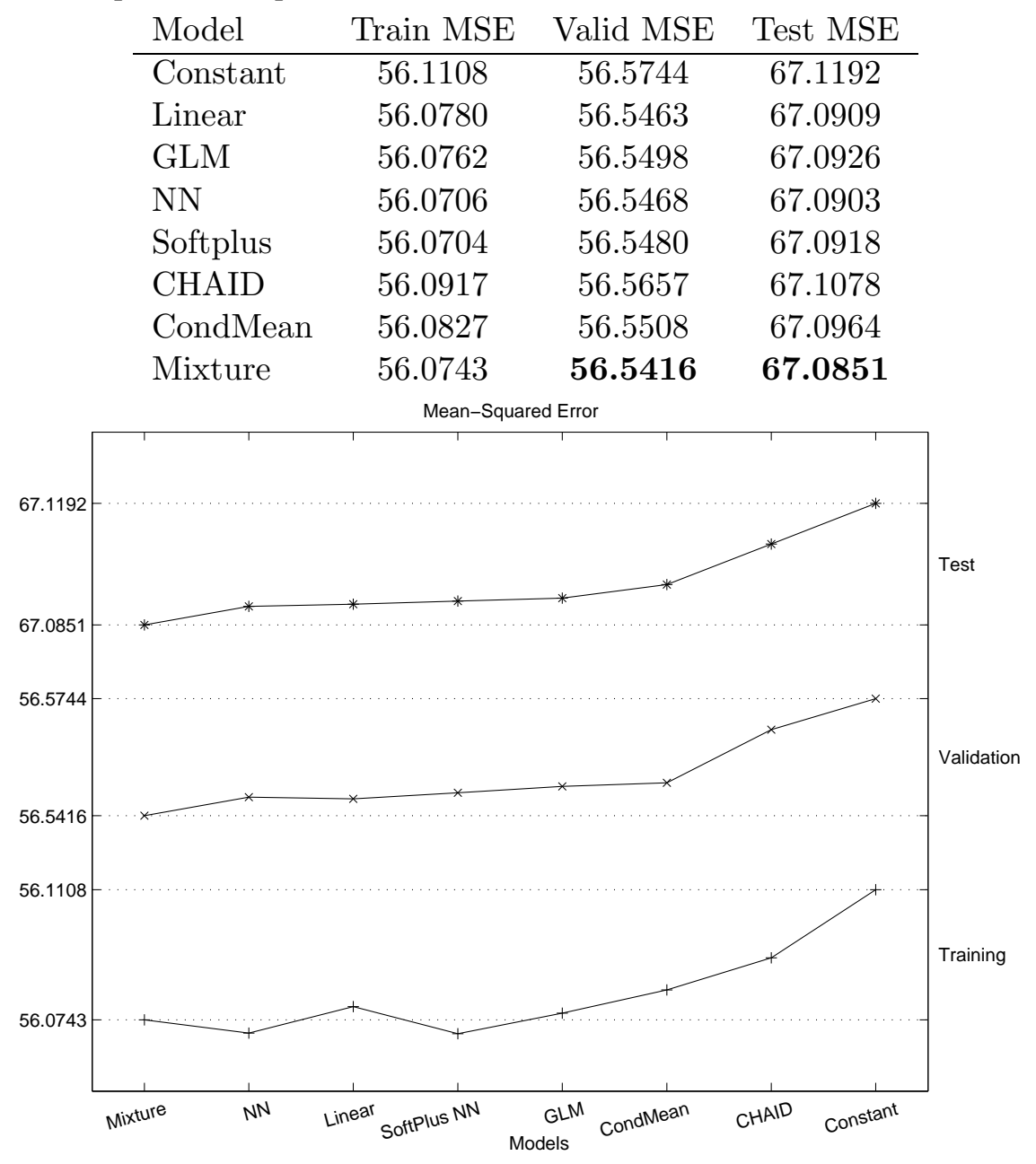

Figure 13. MSE results (from table 1) for eight models . Models have been sorted in ascending order of test results. The training, validation and test curves have been shifted closer together for visualization purposes. The out-of-sample test performance of the mixture model is significantly better than any of the other. Validation based model selection is confirmed on test results. 
the models have been trained using the same input profile variables. For each insurance policy, a total of 33 input variables were used and the total claims for an accident came from five main coverages: bodily injury, accident benefit, property damage, collision and comprehensive. Two other minor coverages were also included: death benefit and loss of use. In the table, $N N$ stands for Neural Network, GLM for Generalized Linear Model, and CondMean for the Greedy Multiplicative Model. The MSE on the training set, validation set and test set are shown for all models. The MSE is with respect to claim amounts and premiums expressed in thousand of dollars. The model with the lowest MSE is the "Mixture model", and it is the model that has been selected for the comparisons with the insurer's current rules for determining insurance premiums to which we shall refer as the Rule-Based Model.

One may wonder from the previous table why the MSE values are so similar across various models for each dataset and much different across the datasets. In particular, all models perform much worse on the testset (in terms of their MSE). There is a very simple explanation. The maximum incurred amount on the test set and on the validation set is around 3 million dollars. If there was one more such large claim in the test set than in the validation set, one would expect the test MSE (calculated for premiums and amounts in thousand of dollars) to be larger by about 7 (these are in units of squared thousand dollars). Thus a difference of 11 can easily be explained by a couple of large claims. This is a reflection of the very thick right-hand tail of the incurred amount distribution (whose standard deviation is only of about 8 thousand dollars). Conversely, this also explains why all MSE are very similar across models for one particular dataset. The MSE values are all mainly driven by very large claims which no model could reliably forecast (no model could lead the insurer to charge a million dollars to a particular insured !) Consequently, truely significant differences between model performances are shadowed by the effect of very large claims on the MSE values. Although the differences between model performance are relatively small, we shall see 
next that careful statistical analysis allows us to discover that some of them are significant.

Figure 13 illustrates graphically the results of the table, with the models ordered according to the validation set MSE. One should note that within each class of models the capacity is tuned according to the performance on the validation set. On the test and validation sets, the Mixture model dominates all the others. Then come the ordinary Neural Network, linear model, and softplus Neural Network. Only slightly worse are the GLM and CondMean (the Greedy Multiplicative model). CHAID fared poorly on this dataset. Note that the CHAID + linear model described in section 5.7 performed worse than ordinary CHAID. Finally, the constant model is shown as a baseline (since it corresponds to assigning the same premium to every 1-year policy). It is also interesting to note from the figure that the model with the lowest training MSE is not necessarily the best out-of-sample (on the validation or test sets). The SVM performance was appalling and is not shown here; it did much worse than the constant model, because it is aiming for the conditional median rather the conditional expectation, which are very different for this kind of data.

Table 2. Statistical Comparison Between Different Learning Models and the Mixture Model. The $\mathrm{p}$-value is for the null hypothesis of no difference between Model \#1 and the best mixture model. Symbols $\hat{\mu}$ and $\hat{\sigma}$ stand for sample mean and standard deviation. Note that ALL differences are statistically significant.

\begin{tabular}{llrrrr} 
Model \#1 & Model \#2 & $\hat{\mu}$ & $\hat{\sigma}$ & $\mathrm{Z}$ & $\mathrm{p}$-value \\
\hline Constant & Mixture & $3.41 \mathrm{e}-02$ & $3.33 \mathrm{e}-03$ & 10.24 & $\mathbf{0}$ \\
Linear & Mixture & $5.82 \mathrm{e}-03$ & $1.32 \mathrm{e}-03$ & 4.41 & $\mathbf{5 . 3 0 e - 0 6}$ \\
GLM & Mixture & $7.54 \mathrm{e}-03$ & $1.15 \mathrm{e}-03$ & 6.56 & $\mathbf{2 . 7 7 e - 1 1}$ \\
NN & Mixture & $5.24 \mathrm{e}-03$ & $1.41 \mathrm{e}-03$ & 3.71 & $\mathbf{1 . 0 3 e - 0 4}$ \\
Softplus & Mixture & $6.71 \mathrm{e}-03$ & $1.09 \mathrm{e}-03$ & 6.14 & $\mathbf{4 . 2 1 e - 1 0}$ \\
CHAID & Mixture & $2.36 \mathrm{e}-02$ & $2.58 \mathrm{e}-03$ & 9.15 & $\mathbf{0}$
\end{tabular}

Table 2 shows a statistical analysis to determine whether the differences in MSE between the Mixture model and each of the other 
models are significant. The Mean column shows the difference in MSE with the Mixture model. The next column shows the Standard Error of that mean. Dividing the mean by the standard error gives $Z$ in the next column. The last column gives the $p$-value of the null hypothesis according to which the true expected squared errors for both models are the same. Conventionally, a value below $5 \%$ or $1 \%$ is interpreted as indicating a significant difference between the two models. The $\mathrm{p}$-values and $\mathrm{Z}$ corresponding to significant differences are highlighted. Therefore the differences in performance between the mixture and the other models are all statistically significant. As mentionned above, the MSE values are very much affected by large claims. Does such a sensitivity to very large claims make statistical comparisons between models incorrect? No. Fortunately all the comparisons are performed on paired data (the squared error for each individual policy), which cancel out the effect of these very large claims (since, for these special cases, the squared error will be huge for all models and of very close magnitude)

Table 3 has similar columns, but it provides a comparison of pairs of models, where the pairs are consecutive models in the order of validation set MSE. What can be seen is that the ordinary Neural Network (NN) is significantly better than the linear model, but the latter, the softplus Neural Network and GLM are not statistically distinguishable. Finally GLM is significantly better than CHAID, which is significantly better than the constant model. Note that although the softplus Neural Network alone is not doing very well here, it is doing very well within the Mixture model (it is the most successful one as a component of the mixture). The reason may be that within the mixture, the parameter estimation for model of the low incurred amounts is not polluted by the very large incurred amounts (which are learned in a separate model).

\subsection{Evaluating Model Fairness}


Table 3. Statistical Comparison Between Pairs of Learning Models. Models are ordered from worst to best. Symbols $\hat{\mu}$ and $\hat{\sigma}$ stand for sample mean and standard deviation. The test is for comparing the sum of MSEs. The p-value is for the null hypothesis of no difference between Model \#1 and Model \#2.

\begin{tabular}{llrrrr} 
Model \#1 & Model \#2 & $\hat{\mu}$ & $\hat{\sigma}$ & $\mathrm{Z}$ & $\mathrm{p}$-value \\
\hline Constant & CHAID & $1.05 \mathrm{e}-02$ & $2.62 \mathrm{e}-03$ & $\mathbf{3 . 9 9}$ & $\mathbf{3 . 2 4 e - 0 5}$ \\
CHAID & GLM & $1.60 \mathrm{e}-02$ & $2.15 \mathrm{e}-03$ & $\mathbf{7 . 4 6}$ & $\mathbf{4 . 2 3 e - 1 4}$ \\
GLM & Softplus & $8.29 \mathrm{e}-04$ & $8.95 \mathrm{e}-04$ & 0.93 & $1.77 \mathrm{e}-01$ \\
Softplus & Linear & $8.87 \mathrm{e}-04$ & $1.09 \mathrm{e}-03$ & 0.82 & $2.07 \mathrm{e}-01$ \\
Linear & NN & $5.85 \mathrm{e}-04$ & $1.33 \mathrm{e}-03$ & 0.44 & $3.30 \mathrm{e}-01$ \\
NN & Mixture & $5.23 \mathrm{e}-03$ & $1.41 \mathrm{e}-03$ & $\mathbf{3 . 7 1}$ & $\mathbf{1 . 0 3 e - 0 4}$
\end{tabular}

Although measuring the predictive accuracy - as done with the MSE in the previous section - is a useful first step in comparing models, it tells only part of the story. A given model could appear significantly better than its competitors when averaging over all customers, and yet perform miserably when restricting attention to a subset of customers.

We consider a model to be fair if different cross-sections of the population are not significantly biased against, compared with the overall population. Model fairness implies that the average premiums within each sub-group should be statistically close to the average incurred amount within that sub-group.

Obviously, it is nearly impossible to correct for any imaginable bias since there are many different criteria to choose from in order to divide the population into subgroups; for instance, we could split according to any single variable (e.g. premium charged, gender, rate group, territory) but also combinations of variables (e.g. all combinations of gender and territory, etc.). Ultimately, by combining enough variables, we end up identifying individual customers, and give up any hope of statistical reliability.

As a first step towards validating models and ensuring fairness, we choose the subgroups corresponding to the location of the deciles of 
the premium distribution. The $i$-th decile of a distribution is the point immediately above $10 i \%$ of the individuals of the population. For example, the 9 -th decile is the point such that $90 \%$ of the population come below it. In other words, the first subgroup contains the $10 \%$ of the customers who are given the lowest premiums by the model, the second subgroup contains the range $10 \%-20 \%$, and so on.

The subgroups corresponding to the Mixture Model (the proposed model) differ slightly from those in the Rule-Based Model (the insurer's current rules for determining insurance premiums). Since the premium distribution for both models is not the same. The subgroups used for evaluating each model are given in Table 4. Since they correspond to the deciles of a distribution, all the subgroups contain approximately the same number of observations $(\approx 28,000$ on the 1998 test set).

Table 4. Subgroups used for evaluating model fairness, for the Mixture and Rule-Based Models. The lowest and highest premiums in the subgroups are given. Each subgroup contains the same number of observations, $\approx$ 28,000 .

\begin{tabular}{l|rrrr} 
& \multicolumn{2}{|c}{ Mixture Model } & \multicolumn{2}{c}{ Rule-Based Model } \\
\cline { 2 - 5 } & \multicolumn{1}{|c}{ Low } & \multicolumn{1}{c}{ High } & \multicolumn{1}{c}{ Low } & \multicolumn{1}{c}{ High } \\
\hline Subgroup 1 & 50.81 & 166.24 & 139.27 & 245.0145 \\
Subgroup 2 & 166.24 & 214.10 & 245.01 & 297.0435 \\
Subgroup 3 & 214.10 & 259.74 & 297.04 & 336.7524 \\
Subgroup 4 & 259.74 & 306.26 & 336.75 & 378.4123 \\
Subgroup 5 & 306.27 & 357.18 & 378.41 & 417.5794 \\
Subgroup 6 & 357.18 & 415.93 & 417.58 & 460.2658 \\
Subgroup 7 & 415.93 & 490.34 & 460.26 & 507.0753 \\
Subgroup 8 & 490.35 & 597.14 & 507.07 & 554.2909 \\
Subgroup 9 & 597.14 & 783.90 & 554.29 & 617.1175 \\
Subgroup 10 & 783.90 & 4296.78 & 617.14 & 3095.7861
\end{tabular}

The bias within each subgroup appears in Figure 14. It shows the average difference between the premiums and the incurred amounts, within each subgroup (recall that the subgroups are divided according 
to the premiums charged by each model, as per Table 4). A positive difference implies that the average premium within a subgroup is higher than the average incurred amount within the same subgroup. $95 \%$ confidence intervals on the mean difference are also given, to assess the statistical significance of the results.

Since subgroups for the two models do not exactly represent the same customers, we shall refrain from directly comparing the two models on a given subgroup. We note the following points:

- For most subgroups, the two models are being fair: the bias is usually not statistically significantly different from zero.

- More rarely, the bias is significantly positive (the models overcharge), but never significantly negative (models undercharge).

- The only subgroup for which both models undercharge is that of the highest-paying customers, the 10-th subgroup. This can be understood, as these customers represent the highest risk; a high degree of uncertainty is associated with them. This uncertainty is reflected in the huge confidence intervals on the mean difference, wide enough not to make the bias significantly different from zero in both cases. (The bias for the Rule-Based Model is nearly significant.)

From these results, we conclude that both models are usually fair to customers in all premium subgroups. A different type of analysis could also be pursued, asking a different question: "In which cases do the Mixture and the Rule-Based Models differ the most?" We address this issue in next section.

\subsection{Comparison with Current Premiums}

For this comparison, we used the best (on the validation set) Mixture model and compare it on the test data of 1998 against the insurer's 


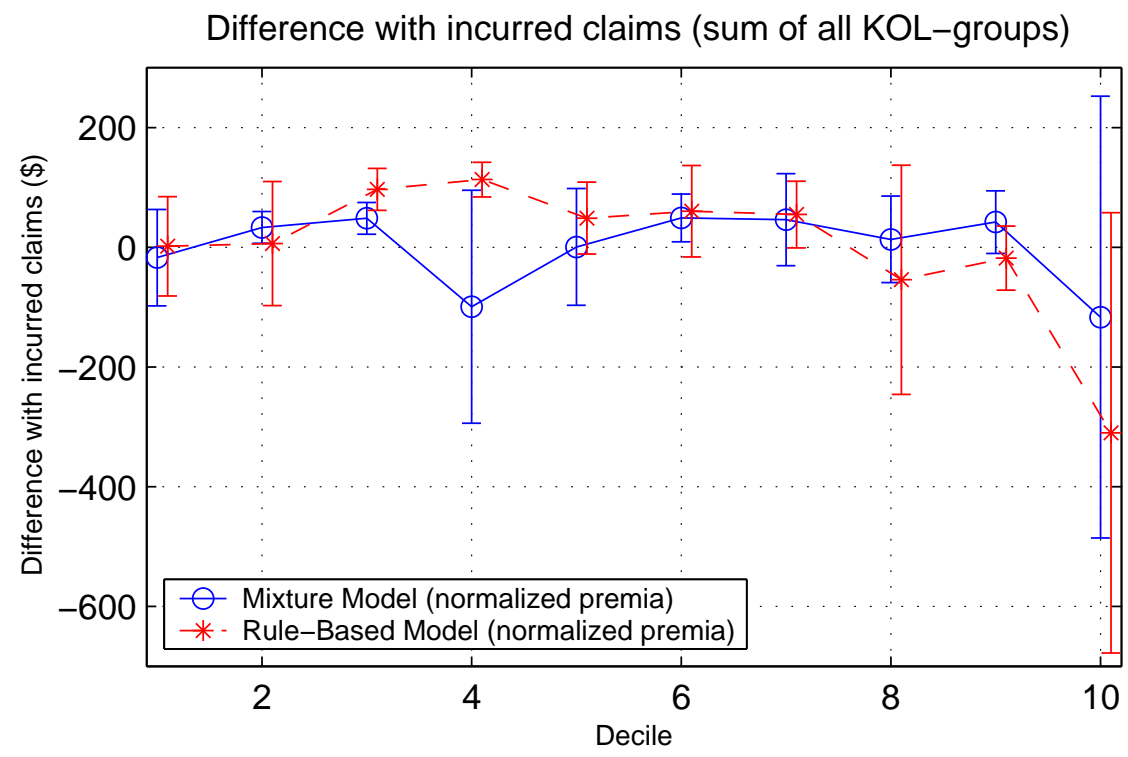

Figure 14. Average difference between premiums and incurred amounts (on the sum over all coverage groups), for the Mixture and Rule-Based models, for each decile of the models' respective premium distribution. We observe that both models are being fair to most customers, except those in the last decile, the highest-risk customers, where they appear to undercharge. The error bars represent $95 \%$ confidence intervals. (Each decile contains $\approx 28,000$ observations.)

Rule-Based Model. Note that for legislative reasons, the Rule-Based Model did not use the same variables as the proposed Mixture Model.

Histograms comparing the distribution of the premiums between the Rule-Based and the Mixture models appear in Figure 15. We observe that the premiums from the Mixture model is smoother and exhibits fatter tails (more probability mass in the right-hand side of the distribution, far from the mean). The Mixture model is better able to recognize risky customers and impose an appropriately-priced premium. 

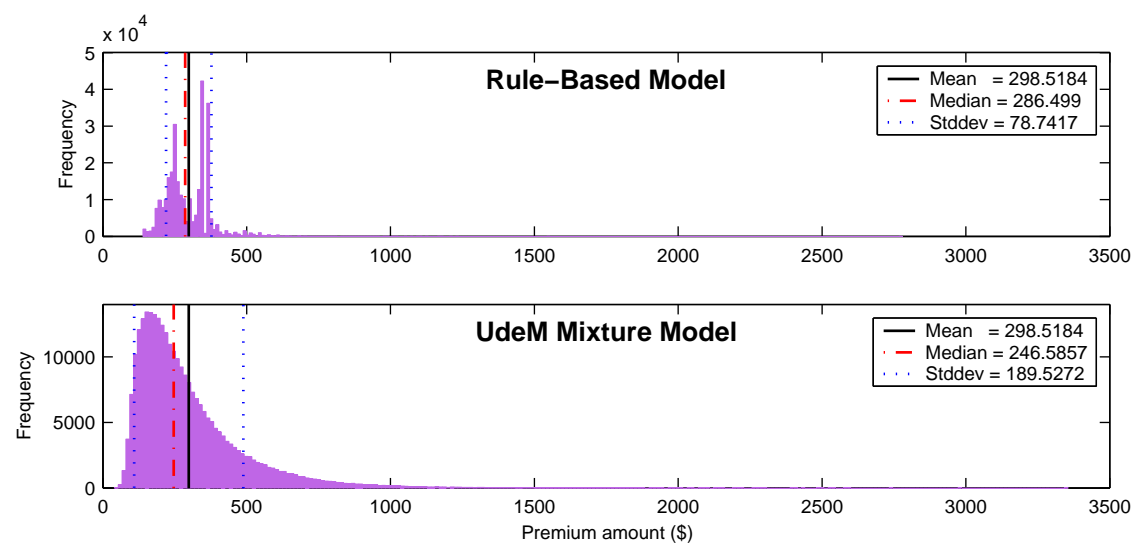

Figure 15. Comparison of the premium distribution for the current RuleBased model and the Mixture model. The distributions are normalized to the same mean. The Mixture model distribution has fatter tails and is much smoother.

This observation is confirmed by looking at the distribution of the premium difference between the Rule-Based and Mixture models, as shown in Figure 16.

We note that this distribution is extremely skewed to the left. This means that for some customers, the Rule-Based model considerably under-charges with respect to the Mixture model. Yet, the median of the distribution is above zero, meaning that the typical customer pays more under the Rule-Based model than under the Mixture model. At the same time, the Mixture model achieves better prediction accuracy, as measured by the Mean-Squared Error (MSE) of the respective models, all the while remaining fair to customers in all categories.

Our overriding conclusion can be stated plainly: the Mixture model correctly charges less for typical customers, and correctly charges more for the "risky" ones. This may be due in part to the use of more variables, and in part to the use of a statistical learning algorithm which is better suited to capturing the dependencies between many 
variables.

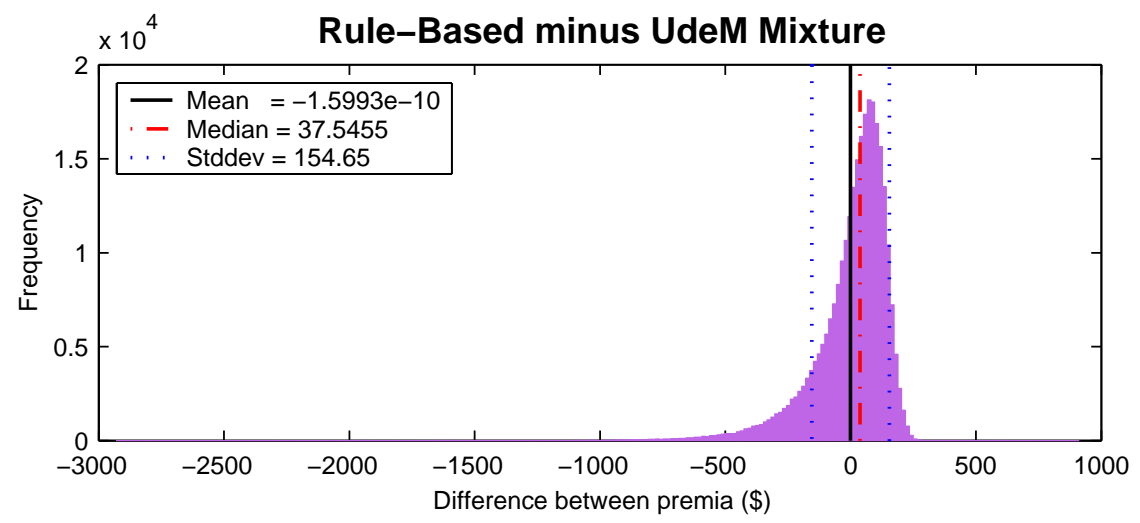

Figure 16. Distribution of the premium difference between the Rule-Based and Mixture models, for the sum of the first three coverage groups. The distribution is negatively skewed: the Rule-Based model severely undercharges for some customers.

\section{Application to Risk Sharing Pool Facilities}

In some provinces and states, improved discrimination between good and bad risks can be used for the purpose of choosing the insureds to be ceeded to risk-sharing pool facilities. In this section, we illustrate the performance of some algorithms when applied to this feat according to the rules that apply in Québec Plan de Répartition des Risques (PRR). According to this facility, an insurer can choose to ceed up to $10 \%$ of its book of business to the pool by paying $75 \%$ of the gross premium that was charged to the insured. Then, in case an accident occurs, the PRR assumes all claim payments. The losses (gains) in the pool are then shared among the insurers in proportion of their market share.

Since automobile insurance is mandatory in Québec, the PRR was 
initially created in order to compensate insurers that were forced by the legislator to insure some risks that had been previously turned down by multiple insurers. The idea was that the insurer could then send these risks to the pool and the losses would be spread among all insurers operating in the province. Of course, such extreme risks represent far less than the allowed $10 \%$ of an insurer's volume. The difference can then be used for other profitable purposes. One possibility is for an insurer to ceed the risks that bring most volatility in the book of business and the pool therefore becomes a means of obtaining reinsurance. In this section, we take a different view: our interest is to use highly discriminative models to identify "wholes" in the ratemaking model, i.e., to identify the risks that have been underpriced the most. Mathematically, this correspond to identifying risks for which the expected value of the claims is higher than $75 \%$ of the gross premium, i.e., those risks with an expected loss ratio of at least $75 \%$, a figure above the industry's average performance. For a particular insurer, the lower the loss ratio, the more difficult it becomes to identify risks that can be (statistically) profitably ceeded. Still, there are a few reasons why important underpricings can be identified:

1. legislation related to ratemaking is more restrictive than the one that pertains to the risk-sharing pool,

2. strategic marketing concerns may have forced the insurer to underprice a certain part of its book of business and,

3. other concerns may not allow the insurer to use highly discriminative models for the purpose of ratemaking.

The last two items can possibly be handled by rule-based systems if the insurer clearly knows which segments of its book of business are underpriced. The legislative context is of more interest to us: stringent legislators refrain insurers from using highly explanatory variables such as sex or age for the purpose of ratemaking. If the pool 
facility ruling is silent in that regard, then underpricings can easily be identified. But this can be done with traditional models.

The interest in highly discriminative models such as Neural Networks comes from the necessity of filing ratemaking plans in a clear fashion. Often, this filing operation limits an actuary in his desire to exploit relevant dependencies between explanatory variables. A lot of insurers still analyze variables independently, in silos, in order to compute individual parameters for each one of them. In that case, no dependency can be captured unless a "home-brewed" variable, resulting from the combination of many, is added. But this is a highly informal procedure which relies on the actuary's thorough knowledge of the problem at hand and technical assistance such as visualization tools. As we have shown in subsection 5.9, Neural Networks are able to automate this procedure and capture the most relevant of these dependencies w.r.t ratemaking. This is where comes in the most important difference between Neural Networks and Generalized Linear Models: automating the detection of depencies.

The superiority of the Neural Network Model is illustrated in Figure 17, where we have simulated the profits that can be generated by an insurer with $100 \mathrm{M} \$$ book of business operating at a global loss ratio of $65 \%$. We compare Neural Networks and Generalized Linear Models as they take turns as ratemaking model and facility model (the model used to identify underpricings in the ratemaking and to choose the risks to be ceeded to the facility). We measured profits as such: for a particular insured risk, let $P_{r}$ and $P_{f}$ be the premiums computed according to the ratemaking and facility models, respectively. Let $C$ be the level of claims that occured for that risk (usually zero). The premiums $P_{r}$ and $P_{f}$ are pure premiums. Since we have assumed a loss ratio of $65 \%$, we can compute the gross premium as $P_{r} / 65 \%$ for the ratemaking model. Then, when a risk is ceeded, the facility keeps $75 \%$ of that premium. So the actual profit of ceeding a particular risk 
is

$$
\text { Actual Profit }=C-75 \% \times P_{r} / 65 \% \text {. }
$$

Similarly, the facility premium $P_{f}$ corresponds to the expected level of claims, so the projected profit of ceeding a risk is:

$$
\text { Projected Profit }=P_{f}-75 \% \times P_{r} / 65 \% \text {. }
$$

Accordingly, the facility premium must be $15.4 \%$ higher than the corresponding ratemaking premium in order to profitably (statistically) ceed a risk. The top graphic of Figure 17 shows that the Neural Network, used as a facility model can help generate substantial profits (between $1.25 \mathrm{M} \$$ and $1.5 \mathrm{M} \$$ for the $100 \mathrm{M} \$$ book of business insurer) when a GLM is used for ratemaking. It profitably identifies underpricings on more than $10 \%$ of the insurer's book of business. Also observe that the difference between the actual and relative profits is relatively small. Since

$$
\text { Actual Profit - Projected Profit }=C-P_{f} \text {, }
$$

we conclude that the Neural Network is very precise at estimating the expected claims level for high risks.

According to the graphic, the insurer has been able to ceed

$$
1.25 \mathrm{M} \$+75 \% \times 10 \% \times 100 \mathrm{M} \$=8.75 \mathrm{M} \$
$$

in claims to the pool. Thus, the ceeded risks had an average loss ratio of $87.5 \%$ up from the global figure of $65 \%$.

On the other hand the second graphic of Figure 17 shows that the GLM model, when used as the facility model mistakingly identifies underpricings in the ratemaking Neural Network model that appear in the projected profit but do not translate in real, actual profit. 

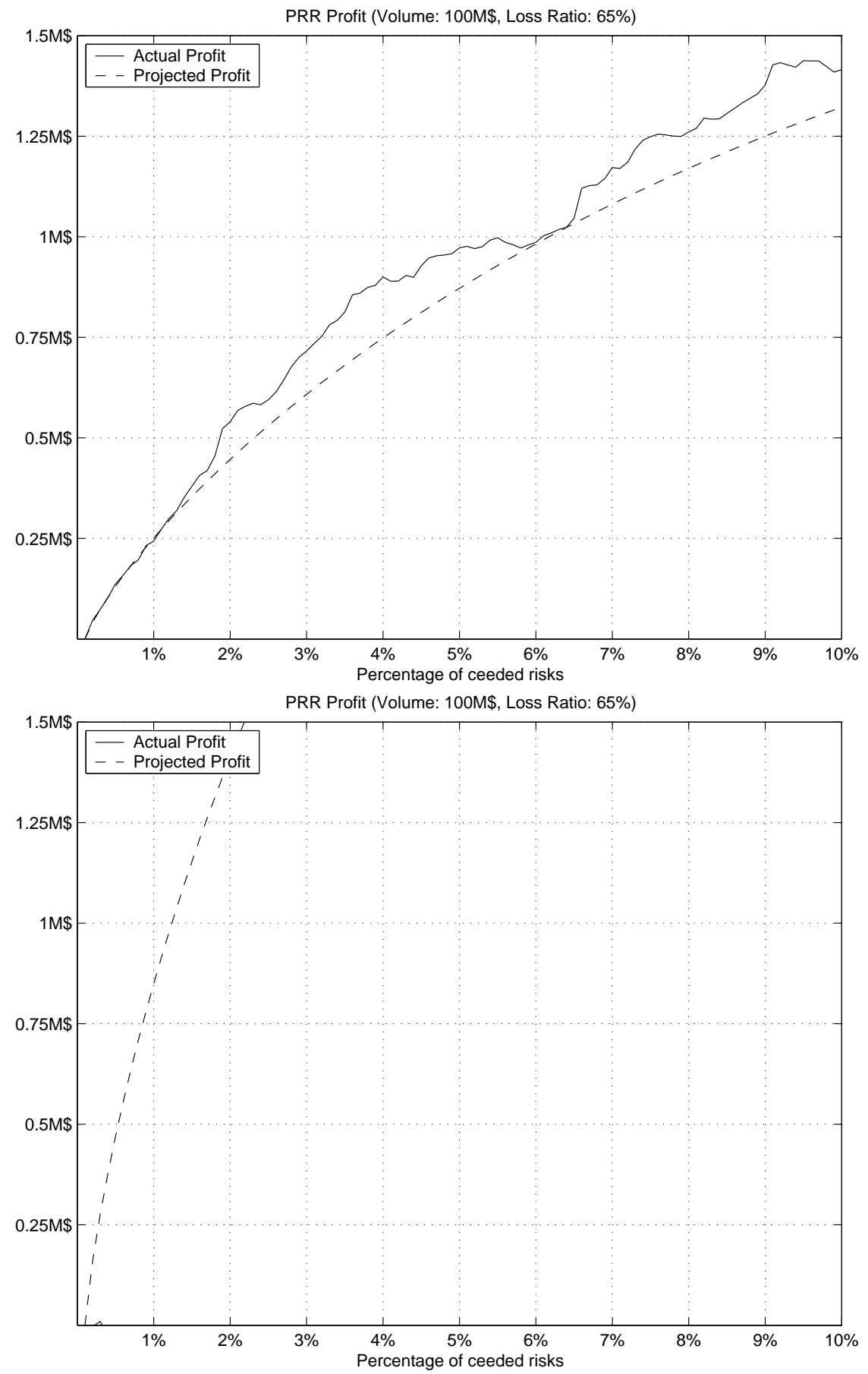

Figure 17. Profit from the PRR facility as a function of the ceeding percentage. Both, the projected profit (dashed) and the actual profit (solid) are shown. These illustrations apply to an insurer with a volume of business of $100 \mathrm{M} \$$ and a global loss ratio of $65 \%$. In the top (bottom) figure, the benchmark model is the Neural Network model (GLM model) and the model used to identify the underpricings is the GLM model (Neural Networks model). 


\section{Conclusion}

Neural networks have been known to perform well in tasks where discrimination is an important aspect of the task at hand and this has lead to many commercially successful application of these modeling tools (Keller 1997). We have shown that, when applied properly while taking into account the particulars of insurance data, that ability to discriminate is also revealed with insurance data. When applied to automobile insurance ratemaking, they allow us to identify more precisely the true risk associated to each insured.

We have argued in favor of the use of statistical learning algorithms such as Neural networks for automobile insurance ratemaking. We have described various candidate models and compared them qualitatively and numerically. We have found that the selected model has significantly outperformed all other models, including the current premium structure. We believe that their superior performance is mainly due to their ability to capture high-order dependencies between variables and to cope with the fat tail distribution of the claims. Other industries have adopted statistical learning algorithms in the last decade and we have shown them to be suited for the automobile insurance industry as well.

Completely changing the rate structure of an insurer can be a costly enterprise, in particular when it involves significant changes in the computer systems handling transactions, or the relations with brokers. We have shown that substantial profit can be obtained from the use of Neural Networks in the context of risk-sharing pools. There are still many other applications where better discrimination of risks can be used profitably, in particular target marketing, fraud detection and elasticity modelling.

\section{Target Marketing:}

When an insurer sends out mail solicitation, only a portion (5\%-10\%) 
of the whole population will be contacted. The goal here is, given this fixed portion, to reach the maximum number of people who will respond positively to the solicitation. Another possibiity would be for the insurer to develop a "customer lifetime value" model that would predict, given an insured's profile, what is the expected present value of the future profits that will be generated by acquiring this particular insured's business. Then, by using the customer lifetime value model in conjunction with a model for the probability of positive response, an insurer could attempt to maximize the profit of its solicitation campaign instead of simply maximizing the number of new insureds.

\section{Fraud Detection}

Fraud represents $10 \%-15 \%$ of all claims. Usually only a portion of the claims will be looked at by an insurer's investigators. The goal of fraud detection is to develop a model that will help an insurer increase the effectiveness of its investigators by referring them cases that are more likely to be fraudulent. In order to do so, one needs a database of previous successful and unsuccessful investigations. Neural Networks have been applied with great success to credit card fraud detection.

\section{Elasticity Modelling}

The greatest benefit from an improved estimation of pure premium derives by considering its application to ratemaking. The main reason for these benefits is that a more discriminant predictor will identify a group of insureds that are significantly undercharged and a (much larger) group that is significantly overcharged. Identifying the undercharged will yield increased profits: increasing their premiums will either directly increase revenues (if they stay) or reduce underwriting losses (if they switch to another insurer). The advantage of identifying the insured profiles which correspond to overcharged premiums can be coupled with a marketing strategy in order attract new customers 
and increase market share, a very powerful engine for increased profitability of the insurer (because of the fixed costs being shared by a larger number of insureds).

To decide on the appropriate change in premium, one also needs to consider market effects. An elasticity model can be independently developped in order to characterize the relation between premium change and the probability of loosing current customers or acquiring new customers. A pure premium model such as the one described in this chapter can then be combined with the elasticity model, as well as pricing constraints (e.g. to prevent too much rate dislocation in premiums, or to satisfy some jurisdiction's regulations), in order to obtain a function that "optimally" chooses for each insured profile an appropriate change in gross premium, in order to maximize a financial criterion.

Clearly, the insurance industry is filled with analytical challenges where better discrimination between good and bad risks can be used profitably. We hope this chapter goes a long way in convincing actuaries to include Neural networks within their set of modeling tools for ratemaking and other analytical tasks.

\section{A Proof of the equivalence of the fairness and precision criterions}

In this section, we show that, when all subpopulations are considered to evaluate fairness, the precision criterion and the fairness criterion, as they were defined in section 3 , both lead to the same premium function.

Theorem 1 The premium function which maximizes precision (in the sense of equation 6) also maximizes fairness (in the sense of equation 9, when all subpopulations are considered), and it is the only one that does maximize it. 


\section{Proof:}

Let $\mathrm{P}$ be a subset of the domain of input profiles. Let $q$ be a premium predictor function. The bias in $\mathrm{P}$ is defined by

$$
b_{q}(P)=\frac{1}{|P|} \sum_{\left(x_{i}, a_{i}\right) \in P}\left(q\left(x_{i}\right)-a_{i}\right) .
$$

Let $F_{q}=-E\left[\sum_{P} b_{q}(P)^{2}\right]$ be the expected "fairness" criterion using premium function $q$, to be maximized (by choosing $q$ appropriately).

Let $p(x)=E[a \mid x]$ be the optimal solution to the precision criterion, i.e. the minimizer of

$$
E\left[(p(X)-A)^{2}\right]
$$

Consider a particular but arbitrary population $P$. Let $q(P)$ denote the average premium for that population using the premium function $q(x)$,

$$
q(P)=\frac{1}{|P|} \sum_{\left(x_{i}, a_{i}\right) \in P} q\left(x_{i}\right)
$$

and similarly, define $a(P)$ the average claim amount for that population,

$$
a(P)=\frac{1}{|P|} \sum_{\left(x_{i}, a_{i}\right) \in P} a_{i}
$$

Then the expected squared bias for that population, using the premium function $q$, is

$$
E\left[b_{q}(P)^{2}\right]=E\left[(q(P)-a(P))^{2}\right]
$$

which is minimized for any $q$ such that $q(P)=E[a(P)]$.

Note in particular that the optimal ESE solution, $p$, is such a minimizer of $F_{q}$, since

$$
p(P)=\frac{1}{|P|} \sum_{\left(x_{i}, a_{i}\right) \in P} E\left[a_{i} \mid x_{i}\right]=E\left[\frac{1}{|P|} \sum_{\left(x_{i}, a_{i}\right) \in P} a_{i}\right]=E[a(P)]
$$


We know therefore that $q=p$ is a minimizer of $F_{q}$, i.e. $\forall q, F_{p} \leq F_{q}$.

Are there other minimizers? Consider a function $q \neq p$, that is a minimizer for a particular population $P_{1}$. Since $q \neq p, \exists x$ s.t. $q(x) \neq$ $p(x)$. Consider the particular singleton population $P_{x}=\{x\}$. On singleton populations, the expected squared bias is the same as the expected squared error. In fact, there is a component of $F$ which contains only the squared biases for the singleton populations, and it is equal to the expected squared error. Therefore on that population (and any other singleton population for which $q \neq p$ ) there is only one minimizer of the expected squared bias, and it is the conditional expectation $p(x)$. So $E\left[(q(X)-A)^{2} \mid X=x\right]>E\left[(p(X)-A)^{2} \mid X=x\right]$ and therefore $E\left[b_{q}\left(P_{x}\right)\right]>E\left[b_{p}\left(P_{x}\right)\right]$. Since $p$ is a maximizer of fairness for all populations, it is enough to prove that $q$ is sub-optimal on one population to prove that the overall fairness of $q$ is less than that of $p$, which is the main statement of our theorem:

$$
\forall q \neq p, F_{q}>F_{p}
$$




\section{References}

Bailey, R. \& Simon, L. (1960). "Two studies in automobile insurance ratemaking", ASTIN Bulletin 1(4): 192-217.

Bellman, R. (1957). Dynamic Programming, Princeton University Press, NJ.

Bengio, Y. \& Gingras, F. (1996). "Recurrent neural networks for missing or asynchronous data", in M. Mozer, D. Touretzky \& M. Perrone (eds), Advances in Neural Information Processing System, Vol. 8, MIT Press, Cambridge, MA, pp. 395-401.

Bishop, C. (1995). Neural Networks for Pattern Recognition, Oxford University Press.

Brown, R. (1988). "Minimum bias with generalized linear models", Proceedings of the Casualty Actuarial Society.

Campbell, J., Lo, A. W. \& MacKinlay, A. (1997). The Econometrics of Financial Markets, Princeton University Press, Princeton.

Chapados, N. \& Bengio, Y. (2003). "Extensions of metric-based model selection", Journal of Machine Learning Research to appear.

Cristianini, N. \& Shawe-Taylor, J. (2000). An Introduction to Support Vector Machines.

Dempster, A. P., Laird, N. M. \& Rubin, D. B. (1977). "Maximumlikelihood from incomplete data via the EM algorithm", Journal of Royal Statistical Society B 39: 1-38.

Diebold, F. X. \& Mariano, R. S. (1995). "Comparing predictive accuracy", Journal of Business and Economic Statistics 13(3): 253263.

Dugas, C., Bengio, Y., Bélisle, F., Nadeau, C. \& Garcia, R. (2001). "A universal approximator of convex functions applied to option 
pricing", Advances in Neural Information Processing Systems, Vol. 13, Denver, CO.

Geman, S., Bienenstock, E. \& Doursat, R. (1992). "Neural networks and the bias/variance dilemma", Neural Computation 4(1): 1-58.

Ghahramani, Z. \& Jordan, M. I. (1994). "Supervised learning from incomplete data via an EM approach", in J. Cowan, G. Tesauro \& J. Alspector (eds), Advances in Neural Information Processing Systems, Vol. 6, Morgan Kaufmann, San Mateo, CA.

Gingras, F., Bengio, Y. \& Nadeau, C. (2000). "On out-of-sample statistics for time-series", Computational Finance 2000.

Hampel, F., Ronchetti, E., Rousseeuw, P. \& Stahel, W. (1986). Robust Statistics, The Approach based on Influence Functions, John Wiley \& Sons.

Hastie, T., Tibshirani, R. \& Friedman, J. (2001). Data Mining, Inference and Prediction, Springer.

Holler, K., Sommer, D. \& Trahair, G. (1999). "Something old, something new in classification ratemaking with a novel use of glms for credit insurance", Casualty Actuarial Society Forum pp. 3184 .

Huber, P. (1982). Robust Statistics, John Wiley \& Sons Inc.

Jacobs, R. A., Jordan, M. I., Nowlan, S. J. \& Hinton, G. E. (1991). "Adaptive mixture of local experts", Neural Computation 3: 7987.

Kass, G. (1980). "An exploratory technique for investigating large quantities of categorical data", Applied Statistics 29(2): 119-127.

Keller, P. E. (1997). Neural networks: Commercial applications. http://www.emsl.pnl.gov:2080/proj/neuron/neural/products. 
McCullagh, P. \& Nelder, J. (1989). Generalized Linear Models, Chapman and Hall, London.

Murphy, K., Brockman, M. \& Lee, P. (2000). "Using generalized linear models to build dynamic pricing systems", Casualty Actuarial Society Forum pp. 107-139.

Nadeau, C. \& Bengio, Y. (2000). "Inference for the generalization error", in S. A. Solla, T. K. Leen \& K.-R. Müller (eds), Advances in Neural Information Processing Systems, Vol. 12, MIT Press, pp. 307-313.

Newey, W. \& West, K. (1987). "A simple, positive semi-definite, heteroscedasticity and autocorrelation consistent covariance matrix", Econometrica 55: 703-708.

Orr, G. \& Müller, K.-R. (1998). Neural Networks: Tricks of the Trade, Springer.

Rousseeuw, P. \& Leroy, A. (1987). Robust Regression and Outlier Detection, John Wiley \& Sons Inc.

Rumelhart, D., Hinton, G. \& Williams, R. (1986). "Learning representations by back-propagating errors", Nature 323: 533-536.

Schölkopf, B., Burges, C. \& Smola, A. (1998). Advances in kernel methods: support vector learning, MIT Press.

Takeuchi, I., Bengio, Y. \& Kanamori, T. (2002). "Robust regression with asymmetric heavy-tail noise distributions", Neural Computation . to appear.

Vapnik, V. (1998). Statistical Learning Theory, John Wiley, Lecture Notes in Economics and Mathematical Systems, volume 454. 